Supplemental materials for:

\title{
The First Total Synthesis of Dragmacidin D
}

Neil K. Garg, Richmond Sarpong, Brian M. Stoltz*

The Arnold and Mabel Beckman Laboratories of Chemical Synthesis, Division of Chemistry and Chemical Engineering, California Institute of Technology, Pasadena, California 91125, USA

Material and Methods. Unless stated otherwise, reactions were performed in flame-dried glassware under a nitrogen or argon atmosphere using dry, deoxygenated solvents. All other commercially obtained reagents were used as received. Solvents were dried by passage through an activated alumina column under argon. Reaction temperatures were controlled by an IKAmag temperature modulator. Thin-layer chromatography (TLC) was performed using E. Merck silica gel 60 F254 precoated plates $(0.25 \mathrm{~mm})$ and visualized by UV or anisaldehyde staining. ICN Silica gel (particle size 0.032-0.063 mm) was used for flash chromatography. Disposable SepPak $\mathrm{C}_{18}$ Vac Cartridges were purchased from Waters and used for all reversed-phase filtrations. HPLC analysis was performed on a Beckman Gold system using a Rainin $\mathrm{C}_{18}$, Microsorb MV, $5 \mathrm{~mm}, 300 \times 4.6 \mathrm{~mm}$ reversed-phased column in $0.1 \%(\mathrm{wt} / \mathrm{v})$ TFA with acetonitrile as eluent and a flow rate of $1.0 \mathrm{~mL} / \mathrm{min}$, gradient elution of $1.25 \%$ acetonitrile $/ \mathrm{min}$. Preparatory reversed-phase HPLC was performed on a Beckman HPLC with a Waters DeltaPak 25 x $100 \mathrm{~mm}, 100 \mathrm{~mm} \mathrm{C}_{18}$ column equipped with a guard, $0.1 \%(\mathrm{wt} / \mathrm{v})$ TFA with acetonitrile as eluent, and gradient elution of $0.50 \%$ acetonitrile/min. For all reversed-phase purifications, water $(18 \mathrm{MW})$ was obtained from a Millipore MiliQ water purification system and TFA from Halocarbon, Inc. ${ }^{1} \mathrm{H}$ and ${ }^{13} \mathrm{C}$ NMR spectra were recorded on either a Varian Mercury 300 (at $300 \mathrm{MHz}$ and $75 \mathrm{MHz}$ respectively), Varian Mercury 500 (at $500 \mathrm{MHz}$ and $125 \mathrm{MHz}$ respectively), or on a Varian Mercury 600 (600 MHz for proton only) spectrometer and are reported relative to $\mathrm{Me}_{4} \mathrm{Si}(\delta$ $0.0)$. Data for ${ }^{1} \mathrm{H}$ NMR spectra are reported as follows: chemical shift ( $\left.\delta \mathrm{ppm}\right)$, multiplicity, 
coupling constant $(\mathrm{Hz})$ and integration. Data for ${ }^{13} \mathrm{C}$ NMR spectra are reported in terms of chemical shift. IR spectra were recorded on a Perkin Elmer Paragon 1000 spectrometer and are reported in frequency of absorption $\left(\mathrm{cm}^{-1}\right)$. UV spectra were measured on a Hewlett-Packard Model 8452A diode array spectrophotometer. High resolution mass spectra were obtained from the UC Irvine Mass Spectral Facility. Analytic chiral HPLC was performed on a chiralcel AD column $(4.6 \mathrm{~mm}$ x $25 \mathrm{~cm})$ obtained from Daicel Chemical Industries, Ltd.

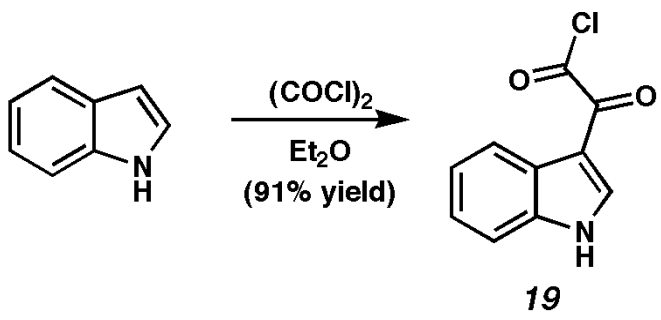

Acid chloride 19. To a solution of indole $(20.0 \mathrm{~g}, 171 \mathrm{mmol})$ in anhydrous diethyl ether $(340 \mathrm{~mL})$ at $0{ }^{\circ} \mathrm{C}$, oxalyl chloride $(17.3 \mathrm{~mL}, 198 \mathrm{mmol})$ was added dropwise over $30 \mathrm{~min}$. The reaction mixture was stirred at $0{ }^{\circ} \mathrm{C}$ for $3 \mathrm{~h}$, then allowed to warm to $23{ }^{\circ} \mathrm{C}$ over $1 \mathrm{~h}$. The resulting yellow crystals were collected by filtration, washed with cold anhydrous ether (100 $\mathrm{mL}$ ), and dried under vacuum to yield 19 (32.52 g, 92\% yield) which was used without further purification.

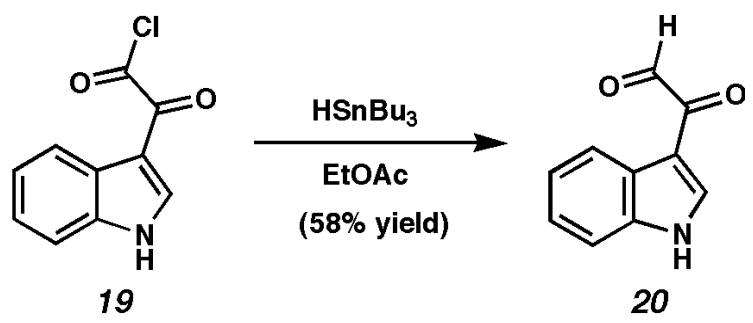

Ketoaldehyde 20. To a suspension of $19(22.0 \mathrm{~g}, 106 \mathrm{mmol})$ in EtOAc $(106 \mathrm{~mL})$ at $0{ }^{\circ} \mathrm{C}$, was added a solution of tributyltin hydride $(28.5 \mathrm{~mL}, 106 \mathrm{mmol})$ in EtOAc $(158 \mathrm{~mL})$. The reaction mixture was stirred at $0{ }^{\circ} \mathrm{C}$ for $30 \mathrm{~min}$, warmed to $23{ }^{\circ} \mathrm{C}$, then stirred for an additional 15 h. Hexane $(150 \mathrm{~mL})$ was added and the resulting yellow powder was collected by filtration. Washing with copious amounts of hexanes $(1 \mathrm{~L})$ and drying under vacuum, gave ketoaldehyde $\mathbf{2 0}$ 
(10.6 g, 58\% yield): $\mathrm{R}_{\mathrm{F}} 0.76$ (13:7 chloroform/methanol eluent); ${ }^{1} \mathrm{H}$ NMR (300 MHz, acetone$\left.\mathrm{d}_{6}\right) \delta 9.54(\mathrm{~s}, 1 \mathrm{H}), 8.65$ (s, 1H), 8.36-8.33 (comp.m, 1H), 7.61-7.58 (comp.m, 1H), 7.33-7.29 (comp.m, 2H); ${ }^{13} \mathrm{C}$ NMR (75 MHz, acetone-d ${ }_{6}$ ) $\delta 192.9,183.2,138.1,125.3,124.6,124.1$, 123.5, 123.1, 113.6, 113.3; IR (film) 3117, 1628, 1580, 1518, $1234 \mathrm{~cm}^{-1}$; HRMS $\left(\mathrm{NH}_{3} \mathrm{CI}\right) \mathrm{m} / \mathrm{z}$ calc'd for $\left[\mathrm{C}_{10} \mathrm{H}_{7} \mathrm{NO}_{2}+\mathrm{H}\right]^{+}:$: 174.0555 , found 174.0555 .

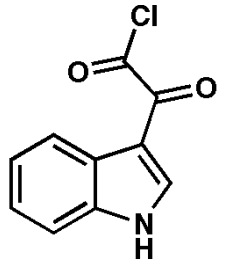

19
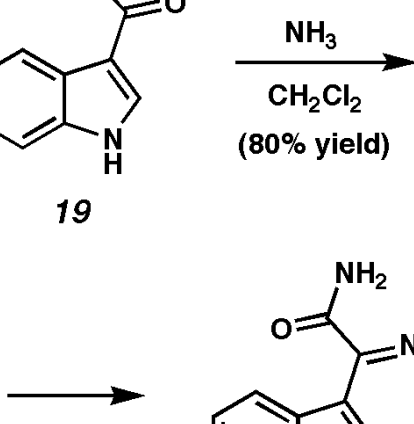

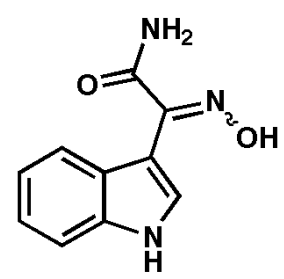

SM2

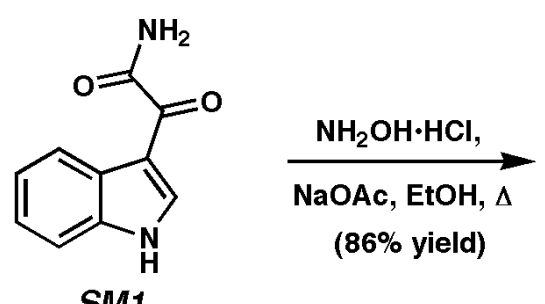

SM1

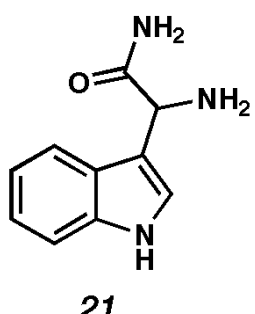

Amino-amide 21. Gaseous ammonia was bubbled through a suspension of 19 (12.4 g, $59.7 \mathrm{mmol})$ in $\mathrm{CH}_{2} \mathrm{Cl}_{2}(300 \mathrm{~mL})$ for $10 \mathrm{~min}$. After stirring for $30 \mathrm{~min}$, the solvent was removed under reduced pressure. Addition of $\mathrm{H}_{2} \mathrm{O}(600 \mathrm{~mL})$ was followed by extraction of the resulting heterogeneous mixture with EtOAc $(2 \times 600 \mathrm{~mL})$. The combined organic layers were washed with brine $(300 \mathrm{~mL})$, dried over magnesium sulfate, and evaporated under reduced pressure to afford the crude amide SM1 (9.0 g, 80\% yield) which was used without further purification.

To a suspension of amide SM1 (500 mg, $5.32 \mathrm{mmol})$ in methanol $(7.8 \mathrm{~mL})$ was added hydroxylamine hydrochloride $(2.0 \mathrm{~g}, 39.9 \mathrm{mmol})$ in $\mathrm{H}_{2} \mathrm{O}(3.8 \mathrm{~mL})$ and sodium acetate $(1.64 \mathrm{~g}$, $39.9 \mathrm{mmol})$ in $\mathrm{H}_{2} \mathrm{O}(3.8 \mathrm{~mL})$. The resulting heterogeneous mixture was heated under reflux for 10 $\mathrm{h}$ and allowed to cool to $23{ }^{\circ} \mathrm{C}$. The solvent was removed under reduced pressure and the remaining crude residue extracted with EtOAc $(3 \times 20 \mathrm{~mL})$. The combined organic layers were washed with brine $(1 \times 20 \mathrm{~mL})$ and dried over sodium sulfate. After removal of solvent under 
reduced pressure, the crude residue was purified by flash chromatography $\left(3: 1 \mathrm{CH}_{2} \mathrm{Cl}_{2} /\right.$ hexanes eluent) to afford oxime SM2 (454 mg, 86\% yield) which was used without further purification.

To a solution of oxime SM2 $(4.07 \mathrm{~g}, 20 \mathrm{mmol})$ in methanol in a stainless steel bomb calorimeter was added $10 \%$ palladium on charcoal $(450 \mathrm{mg})$. The bomb was then purged with hydrogen and pressurized to $450 \mathrm{psi}$. After stirring for $14 \mathrm{~h}$ at $23{ }^{\circ} \mathrm{C}$, the palladium on carbon was removed via filtration and the solvent was removed under reduced pressure. Passage through a plug of silica gel (methanol eluent) afforded the desired aminoamide $\mathbf{2 1}$ (3.5 g, 92\% yield) as a yellow oil: $\mathrm{R}_{\mathrm{F}} 0.10$ (5:1 EtOAc/methanol eluent); ${ }^{1} \mathrm{H}$ NMR (300 MHz, DMSO-d $\left.{ }_{6}\right) \delta 10.93(\mathrm{~s}$, 1H), $7.71(\mathrm{~d}, J=7.7 \mathrm{~Hz}, 1 \mathrm{H}), 7.43(\mathrm{~s}, 1 \mathrm{H}), 7.33(\mathrm{~d}, J=7.7 \mathrm{~Hz}, 1 \mathrm{H}), 7.23(\mathrm{~s}, 1 \mathrm{H}), 7.07-6.93$ (comp.m, 3H), $4.56(\mathrm{~s}, 1 \mathrm{H}), 2.38$ (bs, 2H); ${ }^{13} \mathrm{C}$ NMR (75 MHz, DMSO-d $\left.{ }_{6}\right) \delta 175.9,136.2$, 125.7, 122.8, 120.9, 119.5, 118.2, 116.2, 111.3, 52.5; IR (film) 3176, 1660, $1592 \mathrm{~cm}^{-1}$; HRMS $\left(\mathrm{NH}_{3} \mathrm{CI}\right) \mathrm{m} / z$ calc'd for $\left[\mathrm{C}_{10} \mathrm{H}_{11} \mathrm{~N}_{3} \mathrm{O}+\mathrm{H}\right]^{+}:$190.0980, found 190.0978 .

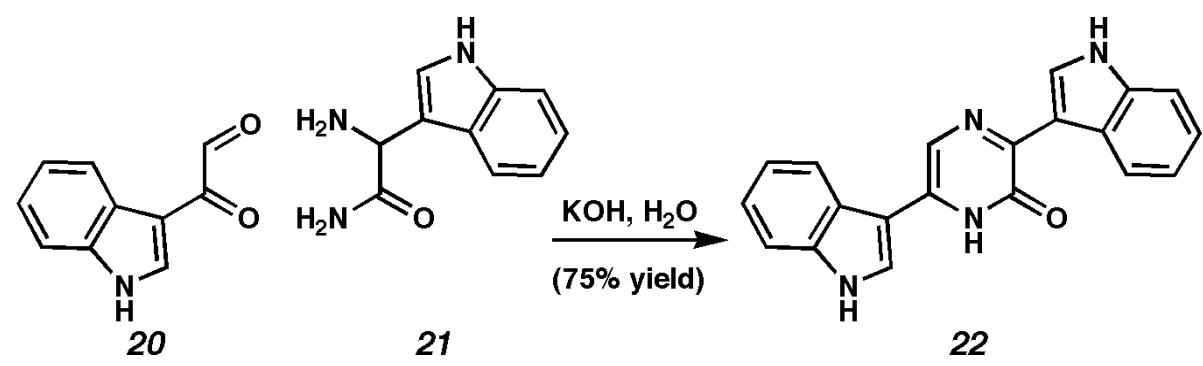

Pyrazinone 22. To a solution of ketoaldehyde 20 (300 mg, $1.73 \mathrm{mmol})$ and aminoamide 21 (321 mg, $1.73 \mathrm{mmol})$ in $\mathrm{H}_{2} \mathrm{O}\left(17 \mathrm{~mL}\right.$ ) at $70{ }^{\circ} \mathrm{C}$ was added powdered potassium hydroxide (487 $\mathrm{mg}, 8.67 \mathrm{mmol}$ ). After stirring at $70{ }^{\circ} \mathrm{C}$ for $5 \mathrm{~h}$, the reaction mixture was allowed to cool to $23{ }^{\circ} \mathrm{C}$, poured into saturated aqueous ammonium chloride $(75 \mathrm{~mL})$, and extracted with EtOAc $(4 \times 75$ $\mathrm{mL}$ ). The combined organic layers were dried briefly over sodium sulfate and concentrated under reduced pressure to afford the desired pyrazinone 22 ( $423 \mathrm{mg}, 75 \%$ yield) as an orange/red solid: $\mathrm{R}_{\mathrm{F}} 0.57$ (5:1 dichloromethane/methanol eluent); ${ }^{1} \mathrm{H}$ NMR (300 MHz, DMSO-d $\left.{ }_{6}\right) \delta 12.23(\mathrm{~s}, 1 \mathrm{H})$, $11.75(\mathrm{~s}, 1 \mathrm{H}), 11.52(\mathrm{~s}, 1 \mathrm{H}), 8.75(\mathrm{~s}, 1 \mathrm{H}), 8.69(\mathrm{~d}, J=7.3 \mathrm{~Hz}, 1 \mathrm{H}), 8.11(\mathrm{~d}, J=2.6 \mathrm{~Hz}, 1 \mathrm{H}$,$) ,$ 8.40-7.82 (comp.m, 2H), 7.51-7.45 (comp.m, 2H), 7.25-7.12 (comp.m, 4H); ${ }^{13} \mathrm{C}$ NMR (125 MHz, DMSO-d $\left.{ }_{6}\right) \delta 155.5,147.2,136.8,136.2,130.1,126.3,125.9,124.1,122.7,122.2,122.0$, 
120.6, 120.1, 119.7, 112.2, 111.9, 111.6, 106.8; IR (film) 3307, 1633, 1602, $1421 \mathrm{~cm}^{-1}$; HRMS (ESI) $m / z$ calc'd for $\left[\mathrm{C}_{20} \mathrm{H}_{15} \mathrm{~N}_{4} \mathrm{O}+\mathrm{Na}\right]^{+}: 349.1065$, found 349.1070 .

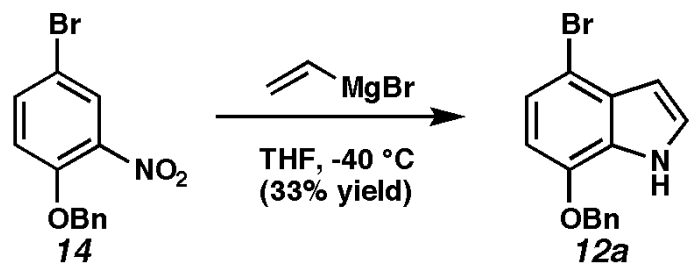

Indole 12a. To a solution of $14(14.0 \mathrm{~g}, 45.5 \mathrm{mmol})$ in $\mathrm{THF}(455 \mathrm{~mL})$ at $-40{ }^{\circ} \mathrm{C}$ was added vinylmagnesium bromide (1.0 M in THF, $159 \mathrm{~mL}, 159 \mathrm{mmol})$ in a dropwise fashion. The reaction mixture was stirred at $-40{ }^{\circ} \mathrm{C}$ for $4 \mathrm{~h}$ and then poured into a saturated aqueous solution of ammonium chloride $(350 \mathrm{~mL})$. The reaction mixture was extracted with ether $(2 \times 200 \mathrm{~mL})$ and the combined organic layers were washed with brine $(1 \times 200 \mathrm{~mL})$, dried over magnesium sulfate, and evaporated under reduced pressure. Dichloromethane $(50 \mathrm{~mL})$ was added and the resulting suspension was filtered over a pad of silica gel topped with celite. Removal of solvent under reduced pressure afforded the crude product as a red oil, which was further purified by flash chromatography (8:1 hexanes/ether eluent) to yield 7-benzyloxy-4-bromoindole 12a (4.54 g, 33\% yield) as a yellow oil: $\mathrm{R}_{\mathrm{F}} 0.30$ (4:1 hexanes/EtOAc eluent); ${ }^{1} \mathrm{H}$ NMR (300 MHz, $\left.\mathrm{CDCl}_{3}\right) \delta 8.46$ (bs, 1H), 7.50-7.40 (comp.m, 5H), 7.20 (d, $J=8.2 \mathrm{~Hz}, 1 \mathrm{H}), 7.16$ (app.t, $J=2.7 \mathrm{~Hz}, 1 \mathrm{H}), 6.61$ 6.58 (comp.m, 2H), 5.17 (s, 2H); ${ }^{13} \mathrm{C}$ NMR (75 MHz, $\left.\mathrm{CDCl}_{3}\right) \delta$ 145.0, 136.8, 129.7, 128.8, 128.5, 128.1, 126.9, 124.4, 122.6, 106.1, 104.4, 103.4, 70.6; IR (film) 3426, $1228 \mathrm{~cm}^{-1}$; HRMS $\left(\mathrm{NH}_{3} \mathrm{CI}\right) \mathrm{m} / z$ calc'd for $\left[\mathrm{C}_{15} \mathrm{H}_{12} \mathrm{BrNO}\right]^{+}:$301.0101, found 301.0102.
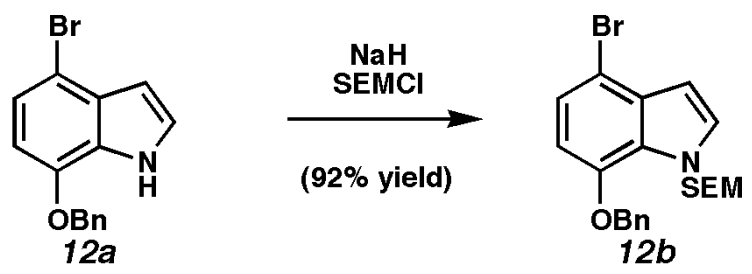

Indole 12b. To a stirred suspension of $\mathrm{NaH}(60 \%$ dispersion in mineral oil, $154 \mathrm{mg}, 4.0$ $\mathrm{mmol})$ in THF $(15 \mathrm{~mL})$ at $0{ }^{\circ} \mathrm{C}$ was added a solution of $\mathbf{1 2 a}(930 \mathrm{mg}, 3.08 \mathrm{mmol})$ in THF (15 $\mathrm{mL}$ ). The reaction mixture was allowed to warm to $23{ }^{\circ} \mathrm{C}$ and stirred for $30 \mathrm{~min}$. The solution 
was cooled to $0{ }^{\circ} \mathrm{C}, \mathrm{SEMCl}(600 \mu \mathrm{L}, 3.4 \mathrm{mmol})$ was added, and the mixture was stirred at $23{ }^{\circ} \mathrm{C}$ for $6 \mathrm{~h}$. The reaction mixture was poured into saturated aqueous ammonium chloride $(20 \mathrm{~mL})$ and extracted with ether $(2 \times 30 \mathrm{~mL})$. The combined organic layers were washed with brine $(1 \mathrm{x}$ $20 \mathrm{~mL}$ ), dried over magnesium sulfate, and evaporated under reduced pressure. The crude residue was purified by flash chromatography (14:1 hexanes/EtOAc eluent) to afford $\mathbf{1 2 b}(1.22 \mathrm{~g}, 92 \%$ yield) as a yellow oil: $\mathrm{R}_{\mathrm{F}} 0.51$ (4:1 hexanes/EtOAc eluent); ${ }^{1} \mathrm{H} \mathrm{NMR}\left(300 \mathrm{MHz}, \mathrm{CDCl}_{3}\right) \delta$ 7.537.50 (comp.m, 2H), 7.46-7.37 (comp. m, 3H), 7.21 (d, $J=3.3 \mathrm{~Hz}, 1 \mathrm{H}), 7.18$ (d, $J=8.2 \mathrm{~Hz}, 1 \mathrm{H}$ ), $6.63(\mathrm{~d}, J=8.2 \mathrm{~Hz}, 1 \mathrm{H}), 6.59$ (d, $J=3.3 \mathrm{~Hz}, 1 \mathrm{H}), 5.73$ (s, 2H), 5.20 (s, 2H), 3.45 (t, $J=8.2 \mathrm{~Hz}$, 2H), $0.84(\mathrm{t}, J=8.2 \mathrm{~Hz}, 2 \mathrm{H}),-0.06(\mathrm{~s}, 9 \mathrm{H}) ;{ }^{13} \mathrm{C} \mathrm{NMR}\left(75 \mathrm{MHz}, \mathrm{CDCl}_{3}\right) \delta 146.3,136.8,131.6$, $129.8,128.8,128.3,127.8,126.2,123.0,106.4,105.7,103.6,77.7,70.8,65.5,17.9,-1.2$; IR (film) 1244, $1054 \mathrm{~cm}^{-1}$; HRMS $\left(\mathrm{NH}_{3} \mathrm{CI}\right) \mathrm{m} / z$ calc'd for $\left[\mathrm{C}_{21} \mathrm{H}_{26} \mathrm{BrNO}_{2} \mathrm{Si}^{+}\right.$: 431.0916, found 431.0919 .
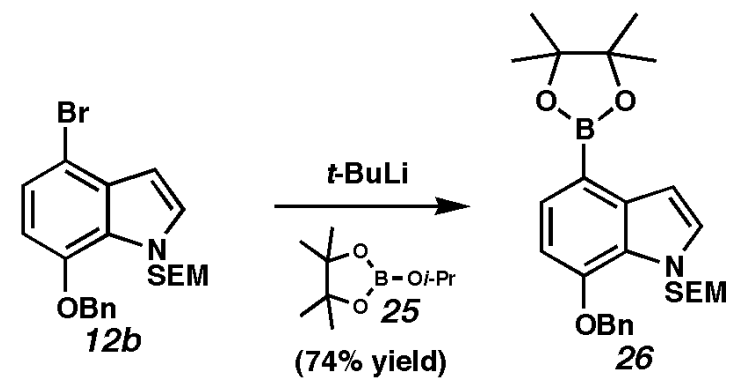

Boronic ester 26. To a solution of $\mathbf{1 2 b}(3.81 \mathrm{~g}, 8.8 \mathrm{mmol})$ in $\mathrm{THF}(147 \mathrm{~mL})$ at $-78{ }^{\circ} \mathrm{C}$ was added $t$-BuLi (1.7 M in pentane, $11.4 \mathrm{~mL}, 19.4 \mathrm{mmol})$. Following addition, the reaction mixture was stirred for $15 \mathrm{~min}$ at $-78{ }^{\circ} \mathrm{C}$ then borane $25(3.6 \mathrm{~mL}, 17.6 \mathrm{mmol})$ was added. The mixture was stirred at $-78{ }^{\circ} \mathrm{C}$ for $1.5 \mathrm{~h}$, allowed to warm to $23{ }^{\circ} \mathrm{C}$, then quenched with saturated aqueous ammonium chloride $(75 \mathrm{~mL})$. The layers were separated and the aqueous portion was extracted with ether $(3 \times 75 \mathrm{~mL})$. The combined organic layers were washed with brine $(100 \mathrm{~mL})$, briefly dried over magnesium sulfate, and evaporated under reduced pressure. The crude residue was purified by flash chromatography (14:1 hexanes/EtOAc eluent) to give boronic ester 26 (3.11 g, $74 \%$ yield) as a yellow oil: $\mathrm{R}_{\mathrm{F}} 0.32\left(9: 1\right.$ hexanes/EtOAc eluent); ${ }^{1} \mathrm{H} \mathrm{NMR}\left(300 \mathrm{MHz}, \mathrm{CDCl}_{3}\right)$ 
$\delta 7.67$ (d, $J=7.7 \mathrm{~Hz}, 1 \mathrm{H}), 7.59-7.56$ (comp.m, 2H), 7.48-7.39 (comp.m, 3H), 7.25 (d, $J=3.3$ $\mathrm{Hz}, 1 \mathrm{H}), 7.12(\mathrm{~d}, J=3.3 \mathrm{~Hz}, 1 \mathrm{H}), 6.83(\mathrm{~d}, J=7.7 \mathrm{~Hz}, 1 \mathrm{H}), 5.81(\mathrm{~s}, 2 \mathrm{H}), 5.28(\mathrm{~s}, 2 \mathrm{H}), 3.49$ (t, $J$ $=8.2 \mathrm{~Hz}, 2 \mathrm{H}), 1.44(\mathrm{~s}, 12 \mathrm{H}), 0.87(\mathrm{t}, J=8.2 \mathrm{~Hz}, 2 \mathrm{H}),-0.02(\mathrm{~s}, 9 \mathrm{H}) ;{ }^{13} \mathrm{C}$ NMR $(75 \mathrm{MHz}$, $\left.\mathrm{CDCl}_{3}\right) \delta 149.3,137.0,136.5,129.9,129.7,128.7,128.1,127.8,125.2,105.1,104.2,83.2,77.5$, 70.3, 65.2, 25.1, 17.9, -1.3; IR (film) 1371, 1330, $1251 \mathrm{~cm}^{-1}$; HRMS $\left(\mathrm{NH}_{3} \mathrm{CI}\right) \mathrm{m} / z$ calc'd for $\left[\mathrm{C}_{27} \mathrm{H}_{38} \mathrm{BNO}_{4} \mathrm{Si}\right]^{+}:$479.2668, found 479.2673.
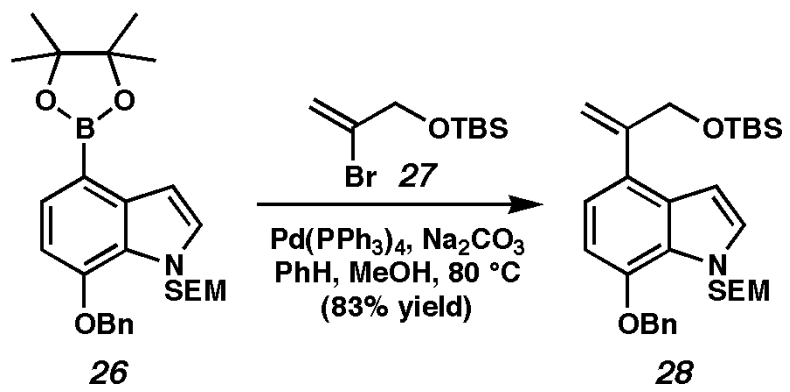

Olefin 28. A solution containing boronic ester $26(3.17 \mathrm{~g}, 6.62 \mathrm{mmol})$ and bromide 27 $(3.32 \mathrm{~g}, 13.2 \mathrm{mmol})$ in benzene $(130 \mathrm{~mL})$, methanol $(30 \mathrm{~mL})$, and aqueous sodium carbonate $(2$ $\mathrm{M}, 11 \mathrm{~mL}$ ) was deoxygenated by bubbling a stream of argon through the reaction mixture for 5 min. Tetrakis(triphenylphosphine)palladium $(0)(1.15 \mathrm{~g}, 0.99 \mathrm{mmol})$ was then added and the flask was equipped with a reflux condenser. The mixture was heated to $80{ }^{\circ} \mathrm{C}$ for $2 \mathrm{~h}$ and allowed to cool to $23{ }^{\circ} \mathrm{C}$. To the reaction vessel was added sodium sulfate $(10 \mathrm{~g})$, which was allowed to stand for $30 \mathrm{~min}$. After filtration over a pad of silica gel $\left(\mathrm{CH}_{2} \mathrm{Cl}_{2}\right.$ eluent) and concentrating to dryness under reduced pressure, the resulting residue was purified by flash chromatography (1:1 $\mathrm{CH}_{2} \mathrm{Cl}_{2} /$ hexanes eluent) to provide olefin $28(2.87 \mathrm{~g}, 83 \%$ yield $)$ as a yellow oil: $\mathrm{R}_{\mathrm{F}} 0.53$ (9:1 hexanes/EtOAc eluent); ${ }^{1} \mathrm{H}$ NMR (300 MHz, $\mathrm{CDCl}_{3}$ ) $\delta$ 7.55-7.53 (comp.m, 2H), 7.46-7.37 (comp.m, 3H), 7.18 (d, $J=3.3 \mathrm{~Hz}, 1 \mathrm{H}), 6.94(\mathrm{~d}, J=7.7 \mathrm{~Hz}, 1 \mathrm{H}), 6.74$ (d, $J=8.2 \mathrm{~Hz}, 1 \mathrm{H}), 6.67$ $(\mathrm{d}, J=3.3 \mathrm{~Hz}, 1 \mathrm{H}), 5.78(\mathrm{~s}, 2 \mathrm{H}), 5.62(\mathrm{~m}, 1 \mathrm{H}), 5.40(\mathrm{~m}, 1 \mathrm{H}), 5.24(\mathrm{~s}, 2 \mathrm{H}), 4.55(\mathrm{~s}, 2 \mathrm{H}), 3.48(\mathrm{t}$, $J=8.2 \mathrm{~Hz}, 2 \mathrm{H}), 0.99$ (s, 9H), 0.85 (t, $J=8.2 \mathrm{~Hz}, 2 \mathrm{H}), 0.15$ (s, 6H), -0.06 (s, 9H); ${ }^{13} \mathrm{C}$ NMR $(75$ $\left.\mathrm{MHz}, \mathrm{CDCl}_{3}\right) \delta 146.9,146.3,137.2,130.0,129.3,128.8,128.2,127.8,126.3,126.0,118.9$, 
111.9, 104.2, 103.0, 77.6, 70.6, 65.6, 65.4, 26.2, 18.7, 18.0, -1.2, -5.1; IR (film) 1250, $1088 \mathrm{~cm}^{-1}$; HRMS (ESI) $m / z$ calc'd for $\left[\mathrm{C}_{30} \mathrm{H}_{45} \mathrm{NO}_{3} \mathrm{Si}_{2}+\mathrm{H}\right]^{+}:$524.3016, found 524.3019.

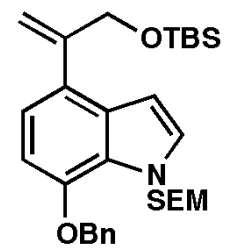

28

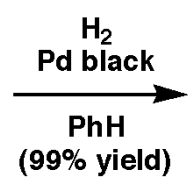

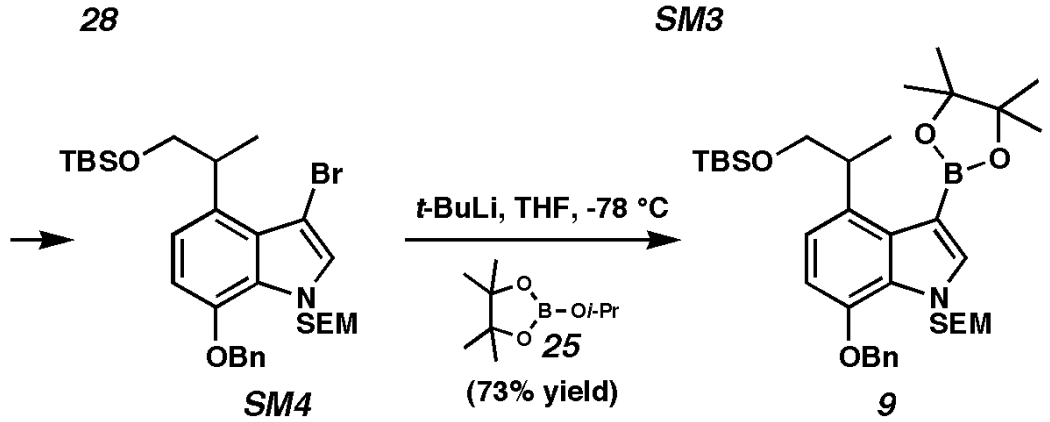

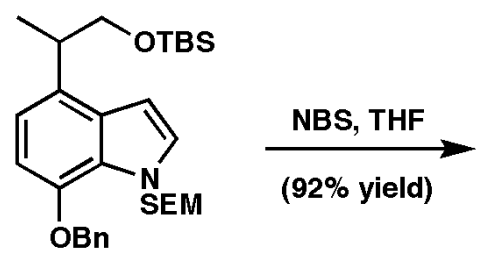

SM3

Boronic ester 9. To a solution of olefin $28(545 \mathrm{mg}, 1.04 \mathrm{mmol})$ in benzene $(12 \mathrm{~mL})$, saturated with hydrogen, was added palladium black $(35 \mathrm{mg}, 0.33 \mathrm{mmol})$. The reaction vessel was purged with hydrogen and kept under a hydrogen atmosphere (1 atm) with vigorous stirring for $1 \mathrm{~h}$. Palladium black was removed via filtration through a pad of silica gel (benzene eluent) to afford the reduced silyl ether SM3 (542 $\mathrm{mg}, 99 \%$ yield) as a yellow oil: $\mathrm{R}_{\mathrm{F}}$ 0.53 (9:1 hexanes/EtOAc eluent); ${ }^{1} \mathrm{H}$ NMR (300 MHz, $\mathrm{CDCl}_{3}$ ) $\delta$ 7.53-7.50 (comp.m, 2H), 7.43-7.34 (comp.m, 3H), 7.16 (d, $J=3.3 \mathrm{~Hz}, 1 \mathrm{H}), 6.86(\mathrm{~d}, J=7.7 \mathrm{~Hz}, 1 \mathrm{H}), 6.71$ (d, $J=7.7 \mathrm{~Hz}, 1 \mathrm{H}), 6.60$ (d, $J=3.3 \mathrm{~Hz}, 1 \mathrm{H}), 5.75$ (s, 2H), 5.20 (s, 2H), 3.87 (dd, $J=9.9,4.9 \mathrm{~Hz}, 1 \mathrm{H}), 3.62$ (app.t, $J=$ $9.3 \mathrm{~Hz}, 1 \mathrm{H}), 3.45$ (t, $J=8.2 \mathrm{~Hz}, 2 \mathrm{H}), 3.30(\mathrm{~m}, 1 \mathrm{H}), 1.40$ (d, $J=6.6 \mathrm{~Hz}, 3 \mathrm{H}), 0.89$ (s, 9H), 0.82 $(\mathrm{t}, J=8.2 \mathrm{~Hz}, 2 \mathrm{H}), 0.00(\mathrm{~s}, 6 \mathrm{H}),-0.09(\mathrm{~s}, 9 \mathrm{H}) ;{ }^{13} \mathrm{C} \mathrm{NMR}\left(75 \mathrm{MHz}, \mathrm{CDCl}_{3}\right) \delta 145.3,137.3$, $130.7,129.5,128.8,128.7,128.1,127.8,125.7,117.3,104.4,101.7,77.6,70.6,69.0,65.4,39.1$, 26.3, 18.7, 18.0, 17.5, -1.1, -4.99, -5.04; IR (film) 1249, $1076 \mathrm{~cm}^{-1}$; HRMS (ESI) $\mathrm{m} / z$ calc'd for $\left[\mathrm{C}_{30} \mathrm{H}_{47} \mathrm{NO}_{3} \mathrm{Si}_{2}+\mathrm{Na}\right]^{+}:$548.2992, found 548.2997.

To a solution of the crude silyl ether SM3 (270 mg, $0.51 \mathrm{mmol})$ in THF $(5 \mathrm{~mL})$, was added $\mathrm{N}$-bromosuccinimide $(922 \mathrm{mg}, 0.51 \mathrm{mmol})$. After stirring for $5 \mathrm{~min}$, the reaction mixture was poured into a saturated aqueous solution of sodium metabisulfite $(3 \mathrm{~mL})$, extracted with 
ether ( $3 \times 2 \mathrm{~mL})$, and dried by passage through a plug of silica gel (ether eluent). After concentrating to dryness under reduced pressure, the crude residue was purified by flash chromatography (1:1 $\mathrm{CH}_{2} \mathrm{Cl}_{2} /$ hexanes eluent) to furnish the 3-bromoindole derivative SM4 (285 mg, $92 \%$ yield) as a yellow oil: $\mathrm{R}_{\mathrm{F}} 0.47\left(1: 1 \mathrm{CH}_{2} \mathrm{Cl}_{2} /\right.$ hexanes eluent); ${ }^{1} \mathrm{H}$ NMR (300 MHz, $\mathrm{CDCl}_{3}$ ) $\delta$ 7.57-7.54 (comp.m, 2H), 7.49-7.40 (comp.m, 3H), 7.24 (s, 1H), 7.02 (d, J = 8.2 Hz, 1H), $6.80(\mathrm{~d}, J=7.7 \mathrm{~Hz}, 1 \mathrm{H}), 5.77$ (d, $J=9.9 \mathrm{~Hz}, 1 \mathrm{H}), 5.73$ (d, $J=10.4 \mathrm{~Hz}, 1 \mathrm{H}), 5.23(\mathrm{~s}, 2 \mathrm{H})$, $4.36(\mathrm{~m}, 1 \mathrm{H}), 4.02$ (dd, $J=9.6,4.7 \mathrm{~Hz}, 1 \mathrm{H}), 3.65$ (dd, $J=9.3,8.3 \mathrm{~Hz}, 1 \mathrm{H}), 3.50$ (t, $J=8.0 \mathrm{~Hz}$, 2H), 1.46 (d, $J=7.1 \mathrm{~Hz}, 3 \mathrm{H}), 0.96(\mathrm{~s}, 9 \mathrm{H}), 0.89$ (t, $J=8.0 \mathrm{~Hz}, 2 \mathrm{H}), 0.08(\mathrm{~s}, 3 \mathrm{H}), 0.06(\mathrm{~s}, 3 \mathrm{H})$, $0.00(\mathrm{~s}, 9 \mathrm{H}) ;{ }^{13} \mathrm{C}$ NMR $\left(75 \mathrm{MHz}, \mathrm{CDCl}_{3}\right) \delta 145.0,136.9,130.1,129.6,128.7,128.2,127.8$, $126.1,125.9,118.7,105.2,90.1,77.7,70.7,69.3,65.6,34.7,26.2,18.6,18.0,-1.1,-5.0,-5.1 ;$ IR (film) $1250 \mathrm{~cm}^{-1}$; HRMS (ESI) $\mathrm{m} / z$ calc'd for $\left[\mathrm{C}_{30} \mathrm{H}_{46} \mathrm{BrNO}_{3} \mathrm{Si}_{2}+\mathrm{Na}\right]^{+}: 626.2097$, found 626.2079 .

To a solution of the 3-bromoindole derivative SM4 (2.5 g, $4.1 \mathrm{mmol})$ in THF (69 mL) at $-78{ }^{\circ} \mathrm{C}$ was added $t$-BuLi $(1.7 \mathrm{M}$ in pentane, $5.4 \mathrm{~mL}, 9.1 \mathrm{mmol})$. The reaction mixture was stirred for $15 \mathrm{~min}$ at $-78{ }^{\circ} \mathrm{C}$ and dioxaborolane $25(1.69 \mathrm{~mL}, 8.3 \mathrm{mmol})$ was added. The mixture was stirred at $-78{ }^{\circ} \mathrm{C}$ for $1 \mathrm{~h}$, quenched with saturated aqueous ammonium chloride $(20 \mathrm{~mL})$, and allowed to warm to $23{ }^{\circ} \mathrm{C}$. The layers were separated and the aqueous layer was extracted with ether $(3 \times 50 \mathrm{~mL})$. The combined organic layers were washed with brine $(75 \mathrm{~mL})$, briefly dried over magnesium sulfate, and concentrated in vacuo. The crude residue was purified by flash chromatography (14:1 hexanes/EtOAc eluent) to afford boronic ester 9 (1.96 g, 73\% yield) as a moderately unstable colorless oil that was used immediately in the coupling reaction that follows: $\mathrm{R}_{\mathrm{F}} 0.38\left(9: 1\right.$ hexanes/EtOAc eluent); ${ }^{1} \mathrm{H}$ NMR $\left(300 \mathrm{MHz}, \mathrm{C}_{6} \mathrm{D}_{6}\right) \delta 7.79(\mathrm{~s}, 1 \mathrm{H}), 7.35$ (d, $J=7.7$ Hz, 1H), 7.20-7.05 (comp.m, 5H), 6.65 (d, $J=8.0 \mathrm{~Hz}, 1 \mathrm{H}), 5.50$ (d, $J=10.6 \mathrm{~Hz}, 1 \mathrm{H}), 5.46$ (d, $J$ $=10.3 \mathrm{~Hz}, 1 \mathrm{H}), 4.86(\mathrm{~s}, 2 \mathrm{H}), 4.70(\mathrm{~m}, 1 \mathrm{H}), 4.09(\mathrm{dd}, J=9.5,4.8 \mathrm{~Hz}, 1 \mathrm{H}), 3.91(\mathrm{dd}, J=9.5,7.3$ Hz, 1H), 3.28 (t, $J=7.7 \mathrm{~Hz}, 2 \mathrm{H}), 1.62(\mathrm{~d}, J=6.6 \mathrm{~Hz}, 3 \mathrm{H}), 1.21$ (s, 6H), 1.19 (s, 6H), 0.98 (s, 9H), 0.64 (t, $J=7.7 \mathrm{~Hz}, 2 \mathrm{H}), 0.00$ (s, 3H), -0.01 (s, 3H), -0.19 (s, 9H). 


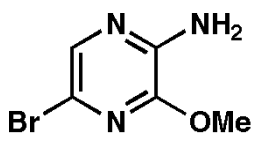

29

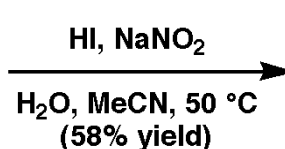

(58\% yield)

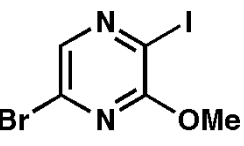

10

Iodopyrazine 10. To a thick-walled flask charged with 29 (100 mg, $0.49 \mathrm{mmol})$ was added acetonitrile (1.0 mL), $\mathrm{H}_{2} \mathrm{O}(1.5 \mathrm{~mL})$, and $48 \%$ aqueous $\mathrm{HI}(1.3 \mathrm{~mL})$. The resulting solution was cooled in an ice bath, and a solution of sodium nitrite $(600 \mathrm{mg}, 8.7 \mathrm{mmol})$ in $\mathrm{H}_{2} \mathrm{O}(1.0 \mathrm{~mL})$ was added in a dropwise fashion. The reaction mixture was sealed, allowed to warm to $23{ }^{\circ} \mathrm{C}$, then stirred at $50{ }^{\circ} \mathrm{C}$ for $30 \mathrm{~h}$. After cooling, the solution was poured into $20 \%$ aqueous $\mathrm{NaOH}$ and extracted with ether $(3 \times 20 \mathrm{~mL})$. The combined organic layers were washed with saturated aqueous sodium metabisulfite $(20 \mathrm{~mL})$ and brine $(20 \mathrm{~mL})$, dried over anhydrous sodium sulfate, then evaporated under reduced pressure. The crude product was then dissolved in a $\mathrm{CH}_{2} \mathrm{Cl}_{2} /$ hexanes mixture (1:1) and filtered over silica gel $\left(1: 1 \mathrm{CH}_{2} \mathrm{Cl}_{2} /\right.$ hexanes eluent $)$ to provide iodopyrazine 10 (90 mg, 58\% yield) as a white powder: $\mathrm{R}_{\mathrm{F}} 0.52\left(1: 1 \mathrm{CH}_{2} \mathrm{Cl}_{2} /\right.$ hexanes eluent); ${ }^{1} \mathrm{H}$ NMR $\left(300 \mathrm{MHz}, \mathrm{CDCl}_{3}\right) \delta 8.07(\mathrm{~s}, 1 \mathrm{H}), 4.05(\mathrm{~s}, 3 \mathrm{H}) ;{ }^{13} \mathrm{C} \mathrm{NMR}\left(75 \mathrm{MHz}, \mathrm{CDCl}_{3}\right) \delta 158.8$, 139.2, 136.1, 104.4, 56.2; IR (KBr) 1357, $1150 \mathrm{~cm}^{-1} ; \mathrm{HRMS}\left(\mathrm{NH}_{3} \mathrm{CI}\right) \mathrm{m} / z$ calc'd for $\left[\mathrm{C}_{5} \mathrm{H}_{4} \mathrm{BrIN}_{2} \mathrm{O}\right]^{+}:$313.8552, found 313.8553 .

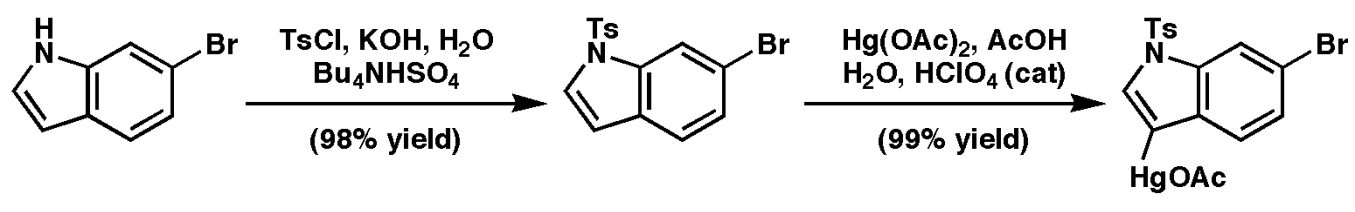

13

SM5

30

Organomercurial 30. To a solution of $13(4.35 \mathrm{~g}, 22.2 \mathrm{mmol})$ in toluene $(22 \mathrm{~mL})$ was added tetrabutylammonium hydrogensulfate $(520 \mathrm{mg}, 1.54 \mathrm{mmol}), \mathrm{KOH}(50 \%$ aqueous solution, $28 \mathrm{~mL})$, and a solution of $p$-toluenesulfonyl chloride $(5.08 \mathrm{~g}, 26.6 \mathrm{mmol})$ in toluene $(44 \mathrm{~mL})$. After stirring for $4 \mathrm{~h}, \mathrm{H}_{2} \mathrm{O}(40 \mathrm{~mL})$ was added and the layers separated. The organic layer was washed with $\mathrm{H}_{2} \mathrm{O}(2 \times 20 \mathrm{~mL})$ and brine $(1 \times 20 \mathrm{~mL})$, dried over magnesium sulfate, and concentrated under reduced pressure to afford 6-bromo- $N$-tosylindole SM5 (7.6 g, 98\% yield) as an off-white powder: $\mathrm{R}_{\mathrm{F}} 0.25$ (9:1 hexanes/EtOAc eluent); ${ }^{1} \mathrm{H}$ NMR (300 $\left.\mathrm{MHz}, \mathrm{CDCl}_{3}\right) \delta 8.19$ 
(s, 1H), 7.75 (d, $J=8.2 \mathrm{~Hz}, 2 \mathrm{H}), 7.53$ (d, $J=3.3 \mathrm{~Hz}, 1 \mathrm{H}), 7.33$ (comp.m, 2H), 7.21 (d, $J=7.7$ $\mathrm{Hz}, 2 \mathrm{H}), 6.61(\mathrm{~d}, J=3.3 \mathrm{~Hz}, 1 \mathrm{H}), 2.32(\mathrm{~s}, 3 \mathrm{H}) ;{ }^{13} \mathrm{C}$ NMR $\left(75 \mathrm{MHz}, \mathrm{CDCl}_{3}\right) \delta$ 145.5, 135.6, 135.1, 130.2, 129.7, 126.9, 126.8, 122.6, 118.3, 116.7, 108.9, 21.7; IR (film) 1364, $1169 \mathrm{~cm}^{-1}$; HRMS $\left(\mathrm{NH}_{3} \mathrm{CI}\right) \mathrm{m} / z$ calc'd for $\left[\mathrm{C}_{15} \mathrm{H}_{12} \mathrm{BrNO}_{2} \mathrm{~S}\right]^{+}:$348.9772, found 348.9773 .

To a solution of 6-bromo- $N$-tosylindole SM5 (7.6 g, $21.7 \mathrm{mmol})$ in acetic acid (145 mL), was added mercuric acetate $(6.92 \mathrm{~g}, 21.7 \mathrm{mmol})$. After stirring at $23{ }^{\circ} \mathrm{C}$ for $15 \mathrm{~min}$, perchloric acid (5 drops) was added. The mixture was stirred for $24 \mathrm{~h}$, poured into $\mathrm{H}_{2} \mathrm{O}(200 \mathrm{~mL})$, then filtered. The resulting white solid was washed with copious amounts of water and dried under vacuum for $12 \mathrm{~h}$ to afford organomercurial derivative 30 (13.05 g, 99\% yield) as an unstable white powder that was used immediately without further purification: $R_{F} 0.57$ (2:1 hexanes/EtOAc eluent); ${ }^{1} \mathrm{H}$ NMR (300 MHz, DMSO-d $\left.{ }_{6}\right) \delta 8.02(\mathrm{~d}, J=1.1 \mathrm{~Hz}, 1 \mathrm{H}), 7.76(\mathrm{~d}, J=8.4 \mathrm{~Hz}, 2 \mathrm{H})$, $7.71(\mathrm{~d}, J=8.4 \mathrm{~Hz}, 1 \mathrm{H}), 7.52$ (s, 1H), $7.42-7.39$ (comp.m, 3H), 2.32 (s, 3H), 1.96 (s, 3H).

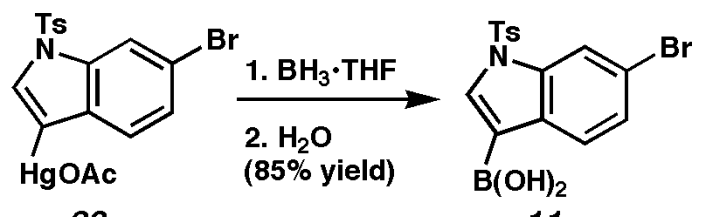

30

11

Boronic acid 11. To a solution of $30(3.91 \mathrm{~g}, 6.4 \mathrm{mmol})$ in THF $(128 \mathrm{~mL})$ at $23{ }^{\circ} \mathrm{C}$ was added borane solution (1M in THF, $32 \mathrm{~mL}, 32 \mathrm{mmol}$ ). The resulting solution was stirred for $1 \mathrm{~h}$, then $\mathrm{H}_{2} \mathrm{O}(38 \mathrm{~mL})$ was added very slowly. After filtration, the organic solvent was evaporated under reduced pressure and the residue was extracted with EtOAc $(2 \times 60 \mathrm{~mL})$. The combined organic layers were washed with brine $(1 \times 30 \mathrm{~mL})$ and concentrated under reduced pressure. Trituration of the crude product with hexanes (4x) afforded boronic acid 11 (2.15 g, 85\% yield) as an unstable off-white solid that was used immediately without further purification.

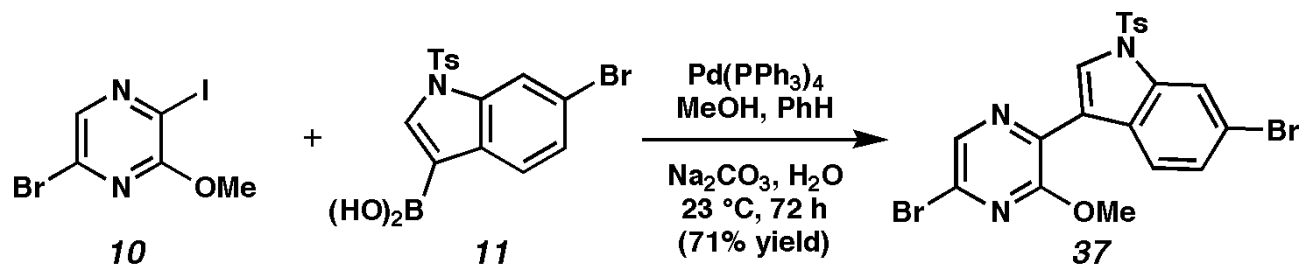


Indolopyrazine 37. A solution containing iodopyrazine 10 (133 $\mathrm{mg}, 0.42 \mathrm{mmol})$ and boronic acid $11(200 \mathrm{mg}, 0.51 \mathrm{mmol})$ in benzene $(10 \mathrm{~mL})$, methanol $(2 \mathrm{~mL})$, and aqueous sodium carbonate $(2 \mathrm{M}, 0.70 \mathrm{~mL})$ was deoxygenated by bubbling a stream of argon through the reaction mixture for $5 \mathrm{~min}$. Tetrakis(triphenylphosphine)palladium(0) $(73 \mathrm{mg}, 0.06 \mathrm{mmol})$ was then added, the flask was evacuated, and purged with $\mathrm{N}_{2}$. The reaction mixture was stirred at $23{ }^{\circ} \mathrm{C}$ for $72 \mathrm{~h}$ and quenched by addition of sodium sulfate $(500 \mathrm{mg})$. Filtration over a pad of silica gel $\left(\mathrm{CH}_{2} \mathrm{Cl}_{2}\right.$ eluent $)$ and concentration to dryness under reduced pressure, followed by trituration of the remaining residue with ether $(3 \mathrm{x})$ and further purification by flash chromatography $\left(\mathrm{CH}_{2} \mathrm{Cl}_{2}\right.$ eluent) afforded indolopyrazine 37 (161 mg, 71\% yield) as an off-white powder: $\mathrm{R}_{\mathrm{F}} 0.13$ (3:1 hexanes/ $\mathrm{CH}_{2} \mathrm{Cl}_{2}$ eluent); ${ }^{1} \mathrm{H}$ NMR $\left(300 \mathrm{MHz}, \mathrm{CDCl}_{3}\right) \delta 8.52(\mathrm{~d}, J=8.2 \mathrm{~Hz}, 1 \mathrm{H}), 8.45(\mathrm{~s}, 1 \mathrm{H})$, $8.33(\mathrm{~s}, 1 \mathrm{H}), 8.18(\mathrm{~d}, J=2.2 \mathrm{~Hz}, 1 \mathrm{H}), 7.81(\mathrm{~d}, J=8.8 \mathrm{~Hz}, 2 \mathrm{H}), 7.43(\mathrm{dd}, J=8.5,2.2 \mathrm{~Hz}, 1 \mathrm{H})$, $7.27(\mathrm{~d}, J=8.8 \mathrm{~Hz}, 2 \mathrm{H}), 4.19(\mathrm{~s}, 3 \mathrm{H}), 2.36(\mathrm{~s}, 3 \mathrm{H}) ;{ }^{13} \mathrm{C} \mathrm{NMR}\left(125 \mathrm{MHz}, \mathrm{CDCl}_{3}\right) \delta 156.5$, $145.9,137.6,137.0,135.8,135.1,132.7,130.4,129.3,128.2,127.6,127.2,125.2,119.3,116.5$, 116.1, 55.2, 21.8; IR (film) 1374, $1165 \mathrm{~cm}^{-1}$; HRMS (ESI) $m / z$ calc'd for $\left[\mathrm{C}_{20} \mathrm{H}_{15} \mathrm{Br}_{2} \mathrm{~N}_{3} \mathrm{O}_{3} \mathrm{~S}+\mathrm{H}\right]^{+}$: 535.9279 , found 535.9272 .
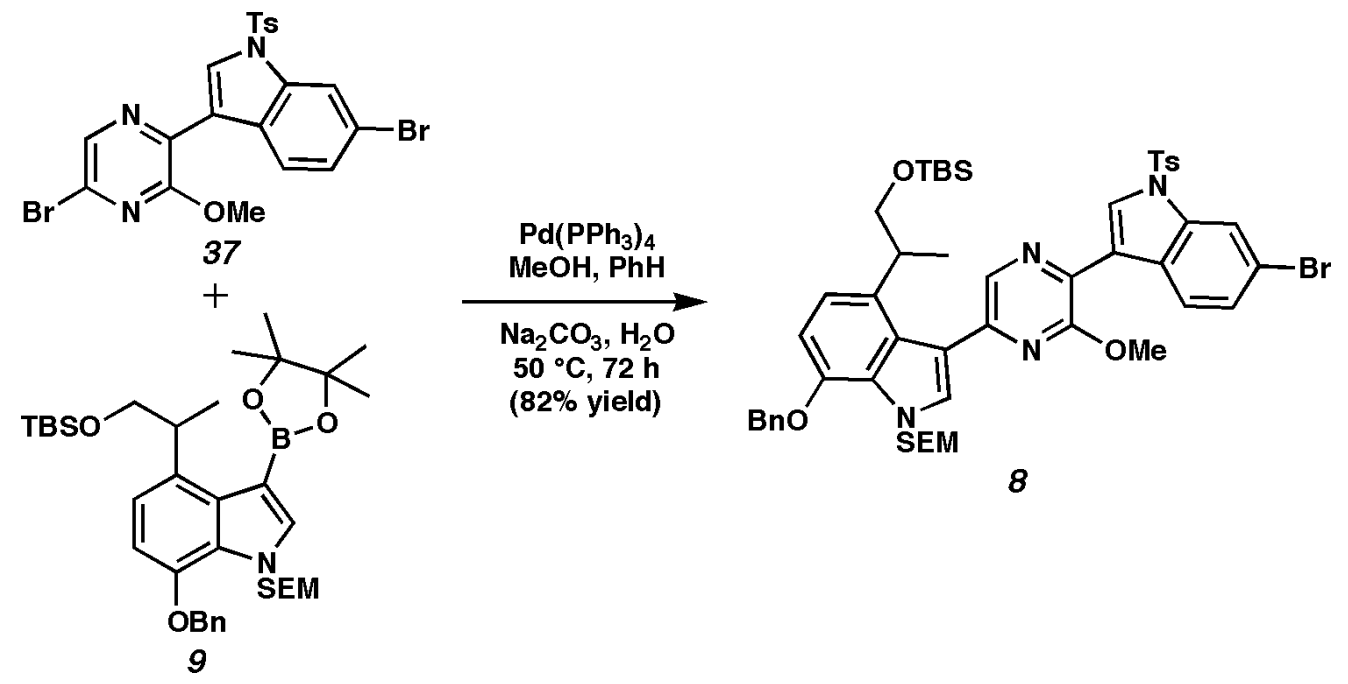

Bis-indole 8. In a Schlenk flask, a solution containing dibromide 37 (82 mg, $0.15 \mathrm{mmol})$ and boronic ester $9(129 \mathrm{mg}, 0.20 \mathrm{mmol})$ in benzene $(4 \mathrm{~mL})$, methanol $(0.80 \mathrm{~mL})$, and aqueous sodium carbonate $(2 \mathrm{M}, 0.25 \mathrm{~mL})$ was deoxygenated by bubbling a stream of argon through the 
reaction mixture for $5 \mathrm{~min}$. Tetrakis(triphenylphosphine)palladium(0) $(27 \mathrm{mg}, 0.02 \mathrm{mmol})$ was then added, the flask was evacuated, purged with $\mathrm{N}_{2}$, and sealed. The reaction mixture was heated to $50{ }^{\circ} \mathrm{C}$ for $72 \mathrm{~h}$, cooled to $23{ }^{\circ} \mathrm{C}$, then quenched by addition of sodium sulfate ( $300 \mathrm{mg}$ ). Following filtration through a pad of silica gel $\left(\mathrm{CH}_{2} \mathrm{Cl}_{2}\right.$ eluent $)$ and evaporation to dryness in vacuo, the remaining residue was purified by flash chromatography $\left(2: 1 \mathrm{CH}_{2} \mathrm{Cl}_{2} /\right.$ hexanes eluent $)$ to give a crude product, which was further purified by flash chromatography (7:1 hexanes/EtOAc eluent) to afford bis-indole 8 (122 mg, $82 \%$ yield) as a yellow oil: $R_{F} 0.2$ (9:1 hexanes/EtOAc eluent); ${ }^{1} \mathrm{H}$ NMR (300 MHz, $\left.\mathrm{CDCl}_{3}\right) \delta 8.71(\mathrm{~d}, J=8.4 \mathrm{~Hz}, 1 \mathrm{H}), 8.52(\mathrm{~s}, 1 \mathrm{H}), 8.50(\mathrm{~s}, 1 \mathrm{H}), 8.27$ $(\mathrm{d}, J=1.8 \mathrm{~Hz}, 1 \mathrm{H}), 7.86(\mathrm{~d}, J=8.4 \mathrm{~Hz}, 2 \mathrm{H}), 7.56-7.38$ (comp.m, 7H), $7.28(\mathrm{~d}, J=8.4 \mathrm{~Hz}, 2 \mathrm{H})$, $7.03(\mathrm{~d}, J=8.1 \mathrm{~Hz}, 1 \mathrm{H}), 6.85(\mathrm{~d}, J=8.4 \mathrm{~Hz}, 1 \mathrm{H}), 5.84(\mathrm{~s}, 2 \mathrm{H}), 5.25(\mathrm{~s}, 2 \mathrm{H}), 4.25$ (s, 3H), 4.07 (m, 1H), 3.62 (dd, $J=9.2,4.4 \mathrm{~Hz}, 1 \mathrm{H}), 3.55$ (t, $J=8.1,2 \mathrm{H}$ ), 3.35 (app.t, $J=9.2 \mathrm{~Hz}, 1 \mathrm{H}$ ), 2.37 (s, 3H), 1.33 (d, $J=7.0 \mathrm{~Hz}, 3 \mathrm{H}), 0.89$ (t, $J=8.4 \mathrm{~Hz}, 2 \mathrm{H}), 0.69$ (s, 9H), -0.04 (s, 9H), -0.16 (s, $3 \mathrm{H}),-0.28(\mathrm{~s}, 3 \mathrm{H}) ;{ }^{13} \mathrm{C} \mathrm{NMR}\left(125 \mathrm{MHz}, \mathrm{CDCl}_{3}\right) \delta 156.1,145.7,145.6,145.4,137.2,135.8$, 135.6, 135.5, 135.1, 131.3, 130.9, 130.3, 128.8, 128.7, 128.3, 127.8, 127.7, 127.3, 127.1, 127.0, 125.5, 119.0, 118.8, 117.3, 116.4, 115.6, 105.5, 78.0, 70.8, 69.2, 65.8, 54.2, 36.8, 25.9, 21.8, 18.2, 18.0, 17.7, -1.2, -5.5, -5.6; IR (film) 1374, 1178, $1087 \mathrm{~cm}^{-1}$; HRMS (ESI) $\mathrm{m} / z$ calc'd for $\left[\mathrm{C}_{50} \mathrm{H}_{61} \mathrm{BrN}_{4} \mathrm{O}_{6} \mathrm{SSi}_{2}+\mathrm{H}\right]^{+}:$981.3112, found 981.3097 .

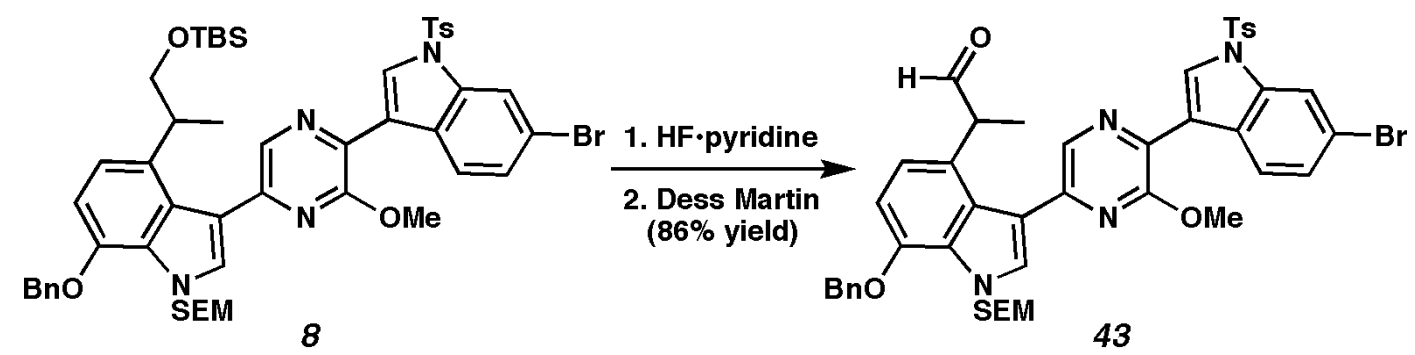

Aldehyde 43. To a Falcon tube containing a THF (5 mL) solution of bis-indole 8 (70 mg, $0.07 \mathrm{mmol})$ at $0{ }^{\circ} \mathrm{C}$, was added $\mathrm{HF} \bullet$ pyridine $(800 \mu \mathrm{L})$ in a dropwise fashion. The reaction mixture was stirred at $0{ }^{\circ} \mathrm{C}$ for $1.5 \mathrm{~h}$ until the reaction was judged complete by TLC. After dilution of the mixture with ether $(10 \mathrm{~mL})$, saturated aqueous sodium bicarbonate $(10 \mathrm{~mL})$ was added in a dropwise manner at $0{ }^{\circ} \mathrm{C}$. The layers were separated and the organic portion was 
further washed with saturated aqueous sodium bicarbonate $(3 \times 10 \mathrm{~mL})$, dried over magnesium sulfate, and concentrated under reduced pressure.

The crude residue prepared above was dissolved in anhydrous $\mathrm{CH}_{2} \mathrm{Cl}_{2}(5 \mathrm{~mL})$ and DessMartin periodinane $(91 \mathrm{mg}, 0.214 \mathrm{mmol})$ was introduced. The reaction mixture was stirred at 23 ${ }^{\circ} \mathrm{C}$ for $20 \mathrm{~min}$, poured into a saturated aqueous solution of sodium bicarbonate/sodium thiosulfate $(1: 1,5 \mathrm{~mL})$, and extracted with $\mathrm{CH}_{2} \mathrm{Cl}_{2}(3 \times 10 \mathrm{~mL})$. The organic layers were washed with brine $(5 \mathrm{~mL})$, dried over magnesium sulfate, and evaporated under reduced pressure to provide the crude product which was purified by flash chromatography $(2: 1$ hexanes/EtOAc) to furnish aldehyde 43 (53 mg, 86\% yield) as a yellow oil: $\mathrm{R}_{\mathrm{F}} 0.67$ (2:1 hexanes/EtOAc eluent); ${ }^{1} \mathrm{H}$ NMR $\left(500 \mathrm{MHz}, \mathrm{CDCl}_{3}\right) \delta 9.73(\mathrm{~s}, 1 \mathrm{H}), 8.69(\mathrm{~d}, J=8.3 \mathrm{~Hz}, 1 \mathrm{H}), 8.52(\mathrm{~s}, 1 \mathrm{H}), 8.46(\mathrm{~s}, 1 \mathrm{H}), 8.24(\mathrm{~d}, J$ $=1.5 \mathrm{~Hz}, 1 \mathrm{H}), 7.86(\mathrm{~d}, J=8.3 \mathrm{~Hz}, 2 \mathrm{H}), 7.56-7.39$ (comp.m, 7H), $7.30(\mathrm{~d}, J=7.8 \mathrm{~Hz}, 2 \mathrm{H}), 6.89$ $(\mathrm{d}, J=8.3 \mathrm{~Hz}, 1 \mathrm{H}), 6.83(\mathrm{~d}, J=8.3 \mathrm{~Hz}, 1 \mathrm{H}), 5.85(\mathrm{~s}, 2 \mathrm{H}), 5.27(\mathrm{~s}, 2 \mathrm{H}), 4.76(\mathrm{q}, J=6.8 \mathrm{~Hz}$, $1 \mathrm{H}), 4.18(\mathrm{~s}, 3 \mathrm{H}), 3.55(\mathrm{t}, J=8.0 \mathrm{~Hz}, 2 \mathrm{H}), 2.39(\mathrm{~s}, 3 \mathrm{H}), 1.37(\mathrm{~d}, J=6.8 \mathrm{~Hz}, 3 \mathrm{H}), 0.89(\mathrm{t}, J=$ $8.0 \mathrm{~Hz}, 2 \mathrm{H}),-0.05(\mathrm{~s}, 9 \mathrm{H}) ;{ }^{13} \mathrm{C} \mathrm{NMR}\left(125 \mathrm{MHz}, \mathrm{CDCl}_{3}\right) \delta 201.9,156.1,146.4,145.7,144.8$, $136.8,136.2$, 135.8, 135.6, 135.0, 131.8, 130.4, 129.1, 128.9, 128.6, 128.5, 128.0, 127.9, 127.5, $127.4,127.2,125.4,124.3,121.0,119.1,116.9,116.5,115.2,105.9,78.2,70.9,66.0,54.3,48.5$, 21.8, 18.0, 15.0, -1.2; IR (film) 1720, 1374, 1177, $1086 \mathrm{~cm}^{-1}$; HRMS (ESI) $\mathrm{m} / z$ calc'd for $\left[\mathrm{C}_{44} \mathrm{H}_{45} \mathrm{BrN}_{4} \mathrm{O}_{6} \mathrm{SSi}+\mathrm{H}\right]^{+}:$865.2090, found 865.2103.

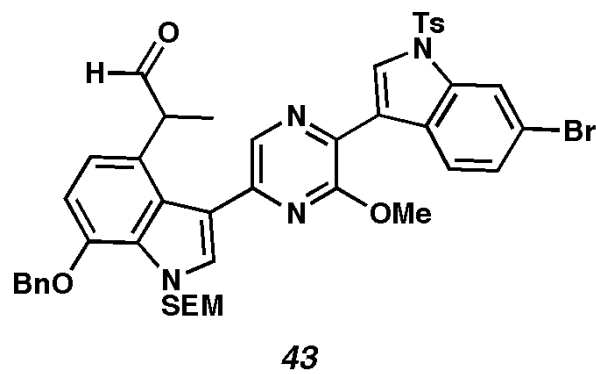

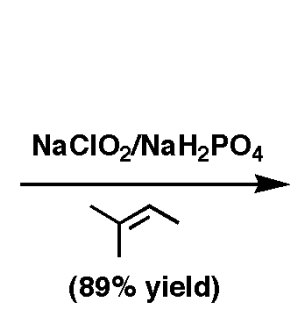

(89\% yield)

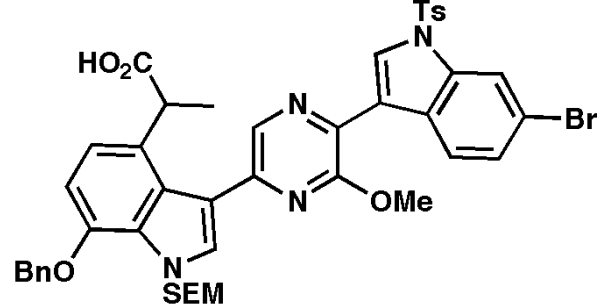

$38 a$

Acid 38a. A solution of aldehyde $43(53 \mathrm{mg}, 0.061 \mathrm{mmol})$ in acetone (12 mL) was treated with a saturated solution of $\mathrm{NaH}_{2} \mathrm{PO}_{4}$ that had been acidified to $\mathrm{pH} 2$ with $1 \mathrm{~N} \mathrm{HCl}(1.4 \mathrm{~mL})$ and cooled to $0{ }^{\circ} \mathrm{C}$. After the addition of 2-methyl-2-butene $(32.5 \mu \mathrm{L}, 0.31 \mathrm{mmol})$, a solution of 
$\mathrm{NaClO}_{2}(13.9 \mathrm{mg}, 0.123 \mathrm{mmol})$ in $\mathrm{H}_{2} \mathrm{O}(1.4 \mathrm{~mL})$ was added dropwise over $5 \mathrm{~min}$. The reaction mixture was poured into cold $\mathrm{H}_{2} \mathrm{O}(2 \mathrm{~mL})$ and extracted with $\mathrm{CH}_{2} \mathrm{Cl}_{2}(2 \times 5 \mathrm{~mL})$. The combined organic layers were dried over magnesium sulfate and evaporated to dryness. The crude residue was passed through a short plug of silica gel (EtOAc eluent) and the solvent was evaporated to afford acid 38a (49 mg, 89\% yield): $\mathrm{R}_{\mathrm{F}} 0.22$ (2:1 hexanes/EtOAc eluent); ${ }^{1} \mathrm{H}$ NMR (300 MHz, $\left.\mathrm{CDCl}_{3}\right) \delta 8.68(\mathrm{~d}, J=8.3 \mathrm{~Hz}, 1 \mathrm{H}), 8.52(\mathrm{~s}, 1 \mathrm{H}), 8.44(\mathrm{~s}, 1 \mathrm{H}), 8.23(\mathrm{~d}, J=1.5 \mathrm{~Hz}, 1 \mathrm{H}), 7.85(\mathrm{~d}, J$ $=8.3 \mathrm{~Hz}, 2 \mathrm{H}), 7.53-7.37$ (comp.m, 7H), $7.28(\mathrm{~d}, J=9.2 \mathrm{~Hz}, 2 \mathrm{H}), 7.08(\mathrm{~d}, J=8.3 \mathrm{~Hz}, 1 \mathrm{H}), 6.84$ $(\mathrm{d}, J=8.3 \mathrm{~Hz}, 1 \mathrm{H}), 5.84(\mathrm{~d}, J=10.3 \mathrm{~Hz}, 1 \mathrm{H}), 5.78(\mathrm{~d}, J=10.3 \mathrm{~Hz}, 1 \mathrm{H}), 5.24(\mathrm{~s}, 2 \mathrm{H}), 4.86(\mathrm{q}, J$ $=6.8 \mathrm{~Hz}, 1 \mathrm{H}), 4.20(\mathrm{~s}, 3 \mathrm{H}), 3.53(\mathrm{t}, J=8.3 \mathrm{~Hz}, 2 \mathrm{H}), 2.36(\mathrm{~s}, 3 \mathrm{H}), 1.42(\mathrm{~d}, J=6.8 \mathrm{~Hz}, 3 \mathrm{H}), 0.86$ (t, $J=8.3 \mathrm{~Hz}, 2 \mathrm{H}),-0.07(\mathrm{~s}, 9 \mathrm{H}) ;{ }^{13} \mathrm{C} \mathrm{NMR}\left(125 \mathrm{MHz}, \mathrm{CDCl}_{3}\right) \delta 178.2,156.2,146.2,145.7$, $145.0,136.9,136.2,135.8,135.7,135.1,131.7,130.4,129.1,128.9,128.6,128.5,128.4,127.9$, $127.5,127.3,127.2,126.3,125.4,119.8,119.1,116.9,116.5,114.9,105.8,78.1,70.9,66.0,54.5$, 40.6, 21.8, 18.4, 18.0, -1.2; IR (film) 2948, 1703, 1373, 1177, 1139, $1088 \mathrm{~cm}^{-1}$; HRMS (ESI) $\mathrm{m} / z$ calc'd for $\left(\mathrm{C}_{44} \mathrm{H}_{45} \mathrm{BrN}_{4} \mathrm{O}_{7} \mathrm{SSi}+\mathrm{Na}\right)^{+}$: 881.2040, found 881.2009.

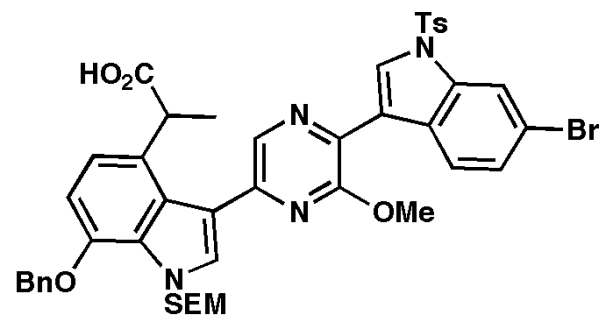
$38 a$

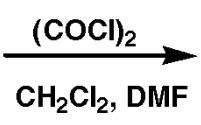

$\mathrm{CH}_{2} \mathrm{Cl}_{2}, \mathrm{DMF}$

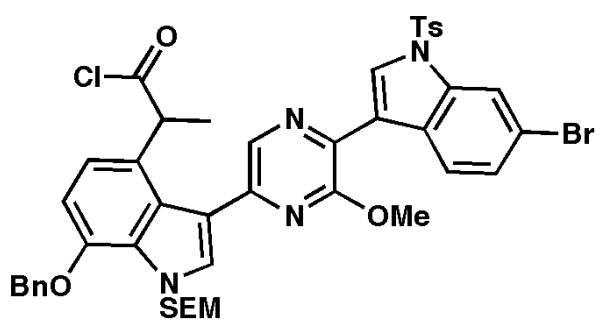

SM6

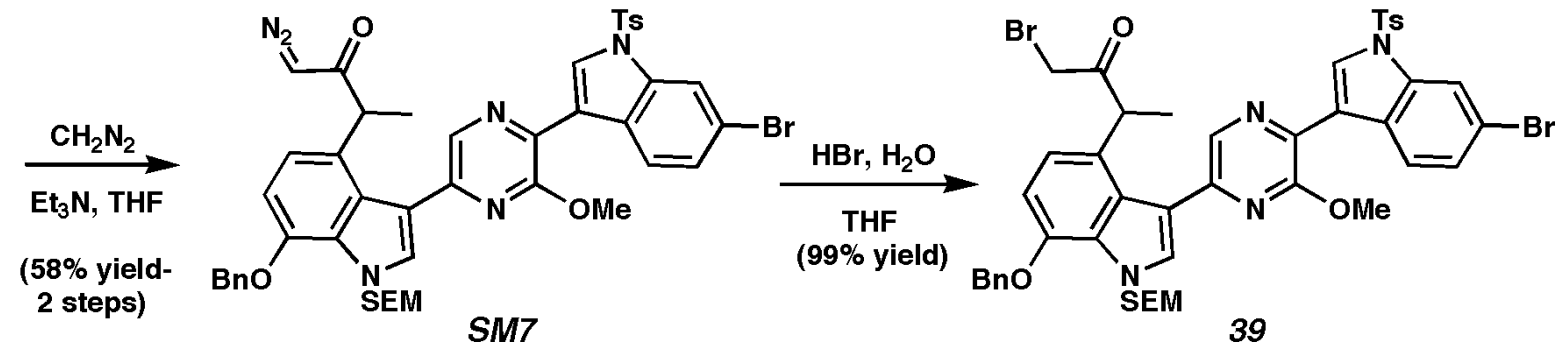

Bromoketone 39. To a solution of carboxylic acid 38a (49 mg, $0.0559 \mathrm{mmol})$ in $\mathrm{CH}_{2} \mathrm{Cl}_{2}$ $(400 \mu \mathrm{L})$ at $0{ }^{\circ} \mathrm{C}$, was added oxalyl chloride $(6.3 \mu \mathrm{L}, 0.0727 \mathrm{mmol})$, followed by DMF $(1 \mu \mathrm{L})$. After stirring at $0{ }^{\circ} \mathrm{C}$ for $30 \mathrm{~min}$, all solvents were removed in vacuo. The crude acid chloride 
SM6 was allowed to dry in vacuo for an additional $1 \mathrm{~h}$ and was used in the next step without further purification.

Note: in the next step diazomethane was dried by storing the ethereal diazomethane solution over potassium hydroxide pellets for $3 \mathrm{~h}$. Immediately before use, the diazomethane was further dried over sodium metal for approximately $15 \mathrm{~min}$. Caution! Diazomethane is toxic and explosive. Caution should always be used when preparing and handling diazomethane, particularly when drying over sodium. All reactions were carried out in a well-ventilated fume hood behind a blast shield. For the use of sodium to dry diazomethane, see: Arndt, F. Org. Synth., 1943, Coll. Vol. 2, 165.

To crude acid chloride SM6 and $\mathrm{Et}_{3} \mathrm{~N}(23 \mu \mathrm{L}, 0.168 \mathrm{mmol})$ in THF $(400 \mu \mathrm{L})$ at $0{ }^{\circ} \mathrm{C}$ was added an ethereal solution of thoroughly dried diazomethane $(1.5 \mathrm{~mL})$ via a flamed glass pipette. The reaction mixture was allowed to warm to $23{ }^{\circ} \mathrm{C}$, poured into saturated aqueous sodium bicarbonate $(1 \mathrm{~mL})$, and extracted with ether $(3 \times 3 \mathrm{~mL})$. The combined organic layers were dried over magnesium sulfate and evaporated under reduced pressure. The crude residue was purified by flash chromatography (4:1 hexanes/EtOAc eluent) to give diazoketone SM7 (29.5 mg, 58\% yield) as a yellow oil.

To SM7 (19 mg, $0.021 \mathrm{mmol})$ in THF $(2 \mathrm{~mL})$ at $0{ }^{\circ} \mathrm{C}, 48 \%$ aqueous $\mathrm{HBr}(50 \mu \mathrm{L})$ was added slowly down the walls of the flask. After stirring for $5 \mathrm{~min}$, the reaction mixture was poured into a saturated aqueous solution of sodium bicarbonate and extracted with ether $(3 \times 4$ $\mathrm{mL}$ ). The combined organic layers were dried over magnesium sulfate and evaporated under reduced pressure to afford bromoketone $39\left(20 \mathrm{mg}, 99 \%\right.$ yield) as a yellow oil: $\mathrm{R}_{\mathrm{F}} 0.68(2: 1$ hexanes/EtOAc eluent); ${ }^{1} \mathrm{H}$ NMR $\left(500 \mathrm{MHz}, \mathrm{CDCl}_{3}\right) \delta 8.69(\mathrm{~d}, J=8.6 \mathrm{~Hz}, 1 \mathrm{H}), 8.53(\mathrm{~s}, 1 \mathrm{H})$, 8.47 (s, 1H), $8.24(\mathrm{~d}, J=1.5 \mathrm{~Hz}, 1 \mathrm{H}), 7.87$ (d, $J=8.2 \mathrm{~Hz}, 2 \mathrm{H}), 7.55-7.38$ (comp.m, 7H), 7.31 $(\mathrm{d}, J=8.2 \mathrm{~Hz}, \mathrm{~d}), 6.85(\mathrm{~d}, 2 \mathrm{H}), 5.83(\mathrm{~s}, 2 \mathrm{H}), 5.25(\mathrm{~s}, 2 \mathrm{H}), 5.01(\mathrm{q}, J=6.7 \mathrm{~Hz}, 1 \mathrm{H}), 4.21(\mathrm{~s}, 3 \mathrm{H})$, $3.76(\mathrm{~d}, J=12.8 \mathrm{~Hz}, 1 \mathrm{H}), 3.67(\mathrm{~d}, J=13.1 \mathrm{~Hz}, 1 \mathrm{H}), 3.55$ (t, $J=8.2 \mathrm{~Hz}, 2 \mathrm{H}), 2.39$ (s, 3H), 1.48 $(\mathrm{d}, J=6.7 \mathrm{~Hz}, 3 \mathrm{H}), 0.88(\mathrm{t}, J=8.1 \mathrm{~Hz}, 2 \mathrm{H}),-0.052(\mathrm{~s}, 9 \mathrm{H}) ;{ }^{13} \mathrm{C}$ NMR $\left(125 \mathrm{MHz}, \mathrm{CDCl}_{3}\right) \delta$ $156.3,146.5,145.8,144.8,136.7,136.5,135.8,135.7,135.1,140.0,130.4,129.2,128.9,128.6$, 
$128.5,128.0,127.7,127.5,127.3,127.2,125.5,125.4,120.7,119.1,116.8,116.5,114.9,106.0$, 78.2, 71.0, 66.1, 54.3, 46.0, 34.2, 21.8, 18.0, 17.8, -1.2; IR (film) 2949, 1724, 1374, 1246, 1178, 1141, $1088 \mathrm{~cm}^{-1}$; HRMS (ESI) $\mathrm{m} / z$ calc'd for $\left(\mathrm{C}_{45} \mathrm{H}_{46} \mathrm{Br}_{2} \mathrm{~N}_{4} \mathrm{O}_{6} \mathrm{SSi}+\mathrm{H}\right)^{+}$: 957.1353, found 957.1376.

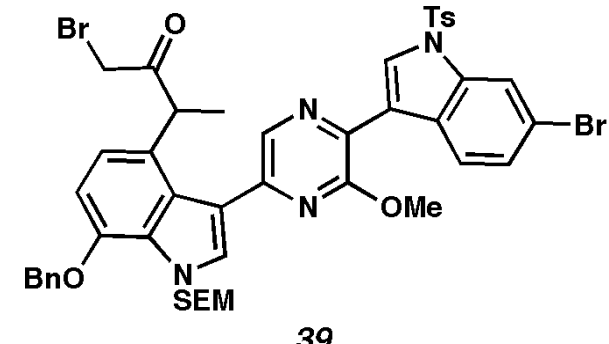

39

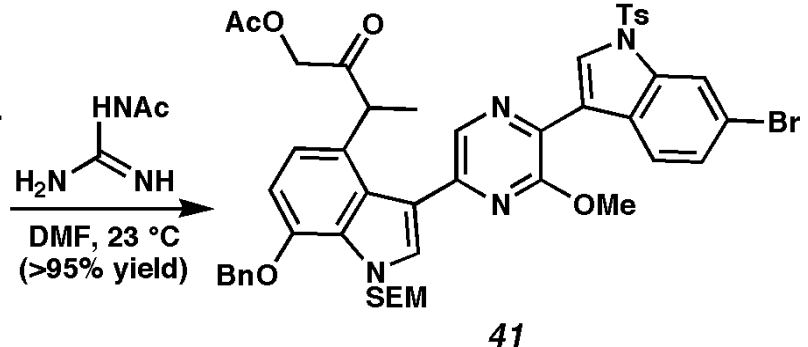

41

Acetoxyketone 41. To a solution of bromoketone 39 (10 mg, $0.0104 \mathrm{mmol})$ in DMF $(350 \mu \mathrm{L})$ was added acetyl guanidine $(32 \mathrm{mg}, 0.316 \mathrm{mmol})$. After stirring at $23{ }^{\circ} \mathrm{C}$ for $48 \mathrm{~h}, \mathrm{H}_{2} \mathrm{O}$ $(1 \mathrm{~mL})$ and EtOAc $(1 \mathrm{~mL})$ were added. The layers were separated and the organic layer was washed with water $(3 \times 500 \mu \mathrm{L})$ and brine $(1 \times 500 \mu \mathrm{L})$, then dried by passage through a plug of silica gel (EtOAc eluent), and evaporated to afford acetoxyketone 41 (9.5 mg, 95\% yield): $R_{F}$ $0.53\left(2: 1\right.$ hexanes/EtOAc eluent); ${ }^{1} \mathrm{H}$ NMR $\left(500 \mathrm{MHz}, \mathrm{CDCl}_{3}\right) \delta 8.69(\mathrm{~d}, J=8.5 \mathrm{~Hz}, 1 \mathrm{H}), 8.54$ (d, $J=0.9 \mathrm{~Hz}, 1 \mathrm{H}), 8.46$ (d, $J=1.2 \mathrm{~Hz}, 1 \mathrm{H}), 8.24(\mathrm{~s}, 1 \mathrm{H}), 7.87$ (d, $J=7.6 \mathrm{~Hz}, 2 \mathrm{H}), 7.55-7.39$ (comp.m, 7H), 7.31 (d, $J=8.2 \mathrm{~Hz}, 2 \mathrm{H}), 6.86$ (s, 2H), 5.83 (s, 2H), 5.25 (s, 2H), 4.83 (q, $J=6.7$ Hz, 1H), 4.47 (s, 2H), 4.21 (s, 3H), 3.54 (t, $J=8.2 \mathrm{~Hz}, 2 \mathrm{H}), 2.39$ (s, 3H), 2.01 (s, 3H), 1.42 (d, $J$ $=7.0 \mathrm{~Hz}, 3 \mathrm{H}), 0.88(\mathrm{t}, J=8.2 \mathrm{~Hz}, 2 \mathrm{H}),-0.05(\mathrm{~s}, 9 \mathrm{H}) ;{ }^{13} \mathrm{C} \mathrm{NMR}\left(125 \mathrm{MHz}, \mathrm{CDCl}_{3}\right) \delta 170.3$, 156.3, 146.4, 145.8, 144.9, 136.8, 136.5, 135.9, 135.6, 135.1, 131.9, 130.4, 129.2, 128.9, 128.6, 128.5, 128.0, 127.6, 127.5, 127.3, 127.2, 125.7, 125.4, 120.6, 119.1, 116.9, 116.5, 114.9, 106.0, 78.2, 71.0, 67.2, 66.1, 54.3, 45.6, 21.9, 20.6, 18.1, 17.3, -1.2; IR (film) 2949, 1750, 1728, 1373, 1244, 1178, 1141, $1088 \mathrm{~cm}^{-1}$; HRMS (ESI) $\mathrm{m} / z$ calc'd for $\left(\mathrm{C}_{47} \mathrm{H}_{49} \mathrm{BrN}_{4} \mathrm{O}_{8} \mathrm{SSi}+\mathrm{H}\right)^{+}$: 937.2302 , found 937.2290 . 

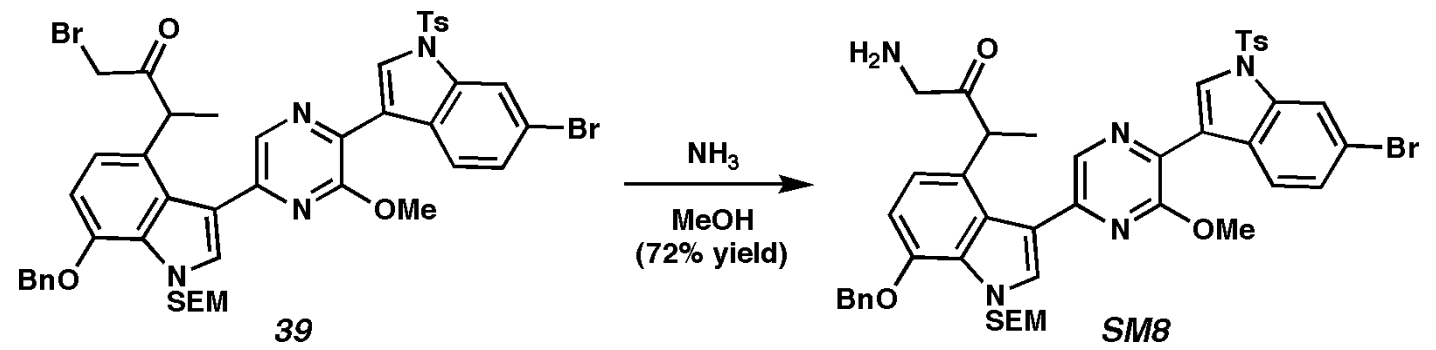

Aminoketone SM8. Bromoketone 39 (6 mg, $0.0062 \mathrm{mmol})$ was dissolved in a saturated solution of ammonia in methanol $(1 \mathrm{~mL})$. After stirring for $6 \mathrm{~h}$ at $23{ }^{\circ} \mathrm{C}$, the reaction mixture was filtered through a plug of silica gel (methanol eluent) and the solvent was evaporated. The crude residue was then purified by preparative thin layer chromatography $\left(7: 1 \mathrm{CH}_{2} \mathrm{Cl}_{2} /\right.$ methanol eluent) to afford aminoketone SM8 (4 mg, 72\% yield): $\mathrm{R}_{\mathrm{F}} 0.67$ (7:1 dichloromethane/methanol eluent); ${ }^{1} \mathrm{H}$ NMR (300 MHz, $\left.\mathrm{CDCl}_{3}\right) \delta 8.19(\mathrm{~d}, 8.8,1 \mathrm{H}), 8.53(\mathrm{~s}, 1 \mathrm{H}), 8.46(\mathrm{~s}, 1 \mathrm{H}), 8.23(\mathrm{~s}, 1 \mathrm{H})$, 7.85 (d, $J=8.4 \mathrm{~Hz}, 2 \mathrm{H}), 7.54-7.37$ (comp.m, 7H), 7.29 (d, $J=8.4 \mathrm{~Hz}, 2 \mathrm{H})$, 6.84-6.83 (comp.m, 2H), $5.82(\mathrm{~s}, 2 \mathrm{H}), 5.24(\mathrm{~s}, 2 \mathrm{H}), 4.71(\mathrm{q}, J=6.6 \mathrm{~Hz}, 1 \mathrm{H}), 4.19(\mathrm{~s}, 3 \mathrm{H}), 3.54(\mathrm{t}, J=8.1 \mathrm{~Hz}, 2 \mathrm{H})$, $3.31(\mathrm{~d}, J=19.3 \mathrm{~Hz}, 1 \mathrm{H}), 3.07(\mathrm{~d}, J=19.1 \mathrm{~Hz}, 1 \mathrm{H}), 2.38(\mathrm{~s}, 3 \mathrm{H}), 1.44$ (d, $J=7.0 \mathrm{~Hz}, 3 \mathrm{H}), 0.87$ (t, $J=8.1 \mathrm{~Hz}, 2 \mathrm{H}),-0.06(\mathrm{~s}, 9 \mathrm{H})$.
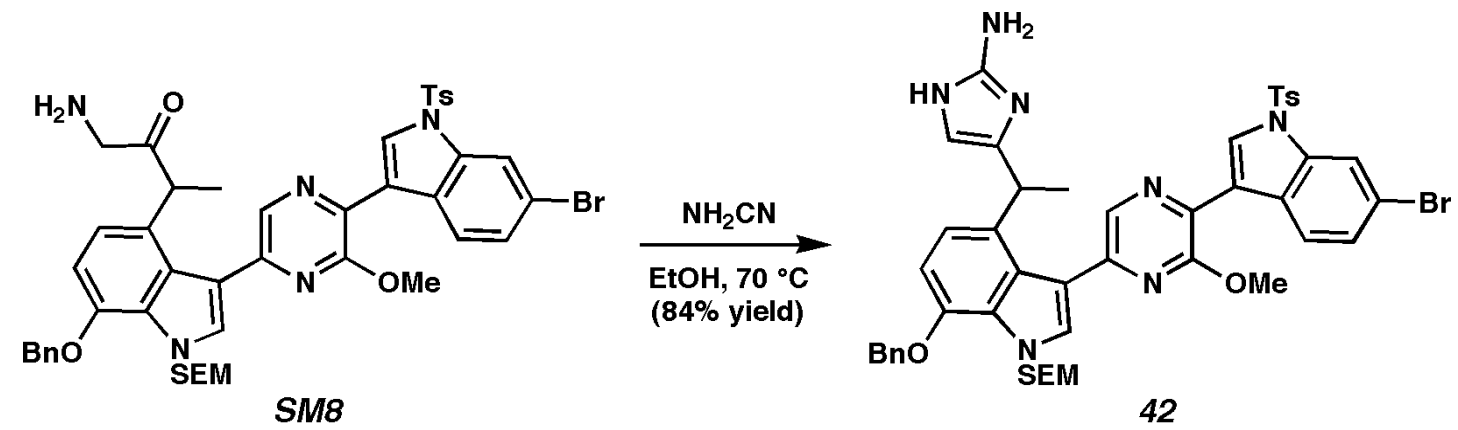

Aminoimidazole 42. To a solution of aminoketone SM8 (7 mg, $0.0078 \mathrm{mmol})$ in ethanol $(700 \mu \mathrm{L})$ was added cyanamide $(15 \mathrm{mg}, 0.36 \mathrm{mmol})$. The reaction vessel was sealed, and heated to $70{ }^{\circ} \mathrm{C}$ for $10 \mathrm{~h}$. After cooling to $23{ }^{\circ} \mathrm{C}$, the reaction mixture was purified by reversedphase filtration through a Sep-Pak column: first 10\% acetonitrile, then $100 \%$ acetonitrile to collect the product. After removal of solvent under reduced pressure, 42 (6 $\mathrm{mg}, 84 \%$ yield) was isolated as an orange/red oil: $\mathrm{R}_{\mathrm{F}} 0.27$ (7:1 dichloromethane/methanol eluent); ${ }^{1} \mathrm{H}$ NMR (500 
$\left.\mathrm{MHz}, \mathrm{CD}_{3} \mathrm{OD}\right) \delta 8.68(\mathrm{~d}, J=8.8 \mathrm{~Hz}, 1 \mathrm{H}), 8.52(\mathrm{~s}, 1 \mathrm{H}), 8.38(\mathrm{~s}, 1 \mathrm{H}), 8.16(\mathrm{~d}, J=1.5 \mathrm{~Hz}, 1 \mathrm{H})$, 7.87 (d, $J=8.1 \mathrm{~Hz}, 2 \mathrm{H}), 7.60-7.33$ (comp.m, 9H), 6.95 (d, $J=8.4 \mathrm{~Hz}, 1 \mathrm{H}), 6.90$ (d, $J=8.4 \mathrm{~Hz}$, $1 \mathrm{H}), 6.10(\mathrm{~s}, 1 \mathrm{H}), 5.85(\mathrm{~s}, 2 \mathrm{H}), 5.28(\mathrm{~s}, 2 \mathrm{H}), 5.09(\mathrm{q}, J=6.8 \mathrm{~Hz}, 1 \mathrm{H}), 4.19(\mathrm{~s}, 3 \mathrm{H}), 3.57(\mathrm{t}, J=$ $7.9 \mathrm{~Hz}, 2 \mathrm{H}), 2.37$ (s, 3H), 1.41 (d, $J=7.0 \mathrm{~Hz}, 3 \mathrm{H}), 0.82$ (t, $J=7.9 \mathrm{~Hz}, 2 \mathrm{H}),-0.10$ (s, 9H); HRMS (ESI) $m / z$ calc'd for $\left(\mathrm{C}_{46} \mathrm{H}_{48} \mathrm{BrN}_{7} \mathrm{O}_{5} \mathrm{SSi}+\mathrm{H}\right)^{+}:$918.2468, found 918.2467.

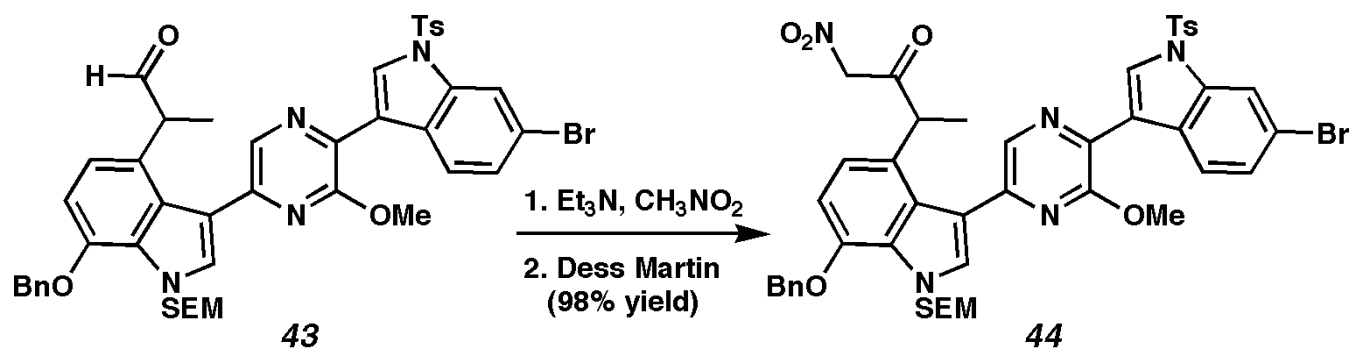

Ketone 44. To aldehyde $43(20 \mathrm{mg}, 0.023 \mathrm{mmol})$ in nitromethane $(1 \mathrm{~mL})$ was added triethylamine $(75 \mu \mathrm{L}, 0.54 \mathrm{mmol})$. The reaction mixture was stirred at $23{ }^{\circ} \mathrm{C}$ for $15 \mathrm{~h}$. The excess nitromethane was removed by evaporation under reduced pressure to afford the crude nitroaldol product which was used without further purification.

The crude residue was dissolved in anhydrous $\mathrm{CH}_{2} \mathrm{Cl}_{2}(1.5 \mathrm{~mL})$ and treated with DessMartin periodinane ( $15 \%$ solution in $\left.\mathrm{CH}_{2} \mathrm{Cl}_{2}, 200 \mu \mathrm{L}, 0.099 \mathrm{mmol}\right)$. The reaction mixture was stirred at $23{ }^{\circ} \mathrm{C}$ for 5 min and quenched by addition of a saturated aqueous solution of sodium bicarbonate/sodium thiosulfate $(1: 1,2 \mathrm{~mL})$. The layers were separated and the aqueous layer was extracted with EtOAc $(8 \times 1 \mathrm{~mL})$. The combined organic layers were washed with brine $(2 \mathrm{~mL})$, dried by passage through a plug of silica gel (EtOAc eluent), and evaporated under reduced pressure to afford ketone 44 (21 mg, 98\% yield) as a yellow oil: $\mathrm{R}_{\mathrm{F}} 0.20$ (3:1 hexanes/EtOAc eluent); ${ }^{1} \mathrm{H}$ NMR (500 MHz, $\mathrm{CDCl}_{3}$ ) $\delta 8.62(\mathrm{~d}, J=8.3 \mathrm{~Hz}, 1 \mathrm{H}), 8.44$ (s, 1H), 8.39 (s, 1H), 8.18 (s, 1H), 7.80 (d, $J=8.3 \mathrm{~Hz}, 2 \mathrm{H}), 7.49-7.33$ (comp.m, 7H), 7.24 (d, $J=8.3 \mathrm{~Hz}, 2 \mathrm{H}), 6.83$ (d, $J=$ $8.3 \mathrm{~Hz}, 1 \mathrm{H}), 6.81(\mathrm{~d}, J=7.8 \mathrm{~Hz}, 1 \mathrm{H}), 5.77$ (s, 2H), $5.21(\mathrm{~s}, 2 \mathrm{H}), 5.03$ (d, $J=14.6 \mathrm{~Hz}, 1 \mathrm{H}), 4.98$ (d, $J=14.6 \mathrm{~Hz}, 1 \mathrm{H}), 4.90(\mathrm{q}, J=6.8 \mathrm{~Hz}, 1 \mathrm{H}), 4.14(\mathrm{~s}, 3 \mathrm{H}), 3.49$ (t, $J=7.8 \mathrm{~Hz}, 2 \mathrm{H}), 2.32$ (s, $3 \mathrm{H}), 1.44(\mathrm{~d}, J=6.8 \mathrm{~Hz}, 3 \mathrm{H}), 0.82(\mathrm{t}, J=8.0 \mathrm{~Hz}, 2 \mathrm{H}),-0.12(\mathrm{~s}, 9 \mathrm{H}) ;{ }^{13} \mathrm{C}$ NMR $(125 \mathrm{MHz}$, $\left.\mathrm{CDCl}_{3}\right) \delta 197.0,156.3,146.9,145.8,144.3,136.9,136.5,135.8,135.6,135.0,132.3,130.4$ 
129.3, 129.0, 128.6, 128.5, 128.0, 127.6, 127.5, 127.4, 127.2, 125.4, 123.7, 121.0, 119.2, 116.7, 116.5, 114.6, 106.2, 82.2, 78.3, 71.0, 66.2, 54.4, 47.6, 21.8, 18.0, 16.9, -1.2; IR (film) 1732, $1559,1376,1178,1080 \mathrm{~cm}^{-1}$.

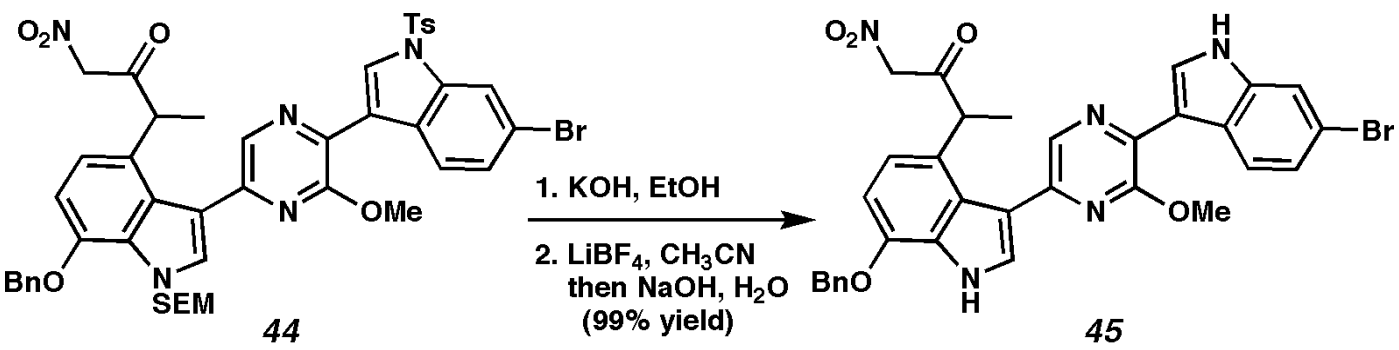

Nitroketone 45. To a suspension of ketone $44(30 \mathrm{mg}, 0.032 \mathrm{mmol})$ in EtOH ( $2 \mathrm{~mL}$, deoxygenated by sparging with argon for $2 \mathrm{~min}$ ), was added powdered $\mathrm{KOH}$ (100 mg, $1.8 \mathrm{mmol}$ ). The reaction vessel was equipped with a reflux condenser and heated to $40{ }^{\circ} \mathrm{C}$ for $2 \mathrm{~h}$. After cooling to $23{ }^{\circ} \mathrm{C}$, the reaction mixture was quenched with saturated aqueous ammonium chloride $(2 \mathrm{~mL})$ and extracted with EtOAc $(8 \times 1 \mathrm{~mL})$. The combined organic layers were washed with brine $(2 \mathrm{~mL})$, dried by passage through a plug of silica gel (EtOAc eluent), and evaporated under reduced pressure to afford the crude detosylated ketone which was used without further purification.

To the crude detosylated ketone prepared above in acetonitrile $(3 \mathrm{~mL}$, deoxygenated by sparging with argon for $2 \mathrm{~min})$ and water $(30 \mu \mathrm{L})$, was added lithium tetrafluoroborate (120 mg, $0.13 \mathrm{mmol})$. The reaction vessel was equipped with a reflux condenser and heated to $70{ }^{\circ} \mathrm{C}$ for $1.5 \mathrm{~h}$ (TLC showed complete consumption of starting material). After cooling to $40{ }^{\circ} \mathrm{C}$, sodium hydroxide ( $20 \%$ aqueous, $2 \mathrm{~mL}$ ) was added. The resulting mixture was stirred for $10 \mathrm{~min}$, allowed to cool to $23{ }^{\circ} \mathrm{C}$, quenched with saturated aqueous ammonium chloride $(2 \mathrm{~mL})$, and extracted with EtOAc $(8 \times 1 \mathrm{~mL})$. The combined organic layers were washed with brine $(2 \mathrm{~mL})$, dried by passage through a plug of silica gel (EtOAc eluent), and evaporated under reduced pressure to afford nitroketone $45\left(20.5 \mathrm{mg}, 99 \%\right.$ yield) as a yellow oil: $\mathrm{R}_{\mathrm{F}} 0.59$ (1:1 hexanes/EtOAc eluent); ${ }^{1} \mathrm{H}$ NMR (300 MHz, acetone- $\left.{ }_{6}\right) \delta 11.14$ (bs, $\left.1 \mathrm{H}\right), 10.86(\mathrm{bs}, 1 \mathrm{H}), 8.82$ (d, $J=8.8 \mathrm{~Hz}, 1 \mathrm{H}), 8.54$ (s, 1H), 8.43 (m, 1H), 7.75-7.72 (comp.m, 2H), 7.62-7.59 (comp.m, 
2H), 7.47-7.30 (comp.m, 4H), 6.95 (d, $J=8.1 \mathrm{~Hz}, 1 \mathrm{H}), 6.85$ (d, $J=8.1 \mathrm{~Hz}, 1 \mathrm{H}), 5.41-5.22$ (comp.m, 5H), 4.19 (s, 3H), 1.47 (d, $J=7.0 \mathrm{~Hz}, 3 \mathrm{H}) ;{ }^{13} \mathrm{C}$ NMR (125 MHz, acetone-d $\left.{ }_{6}\right) \delta 198.7$, 156.2, 146.3, 143.6, 139.4, 138.6, 138.3, 136.1, 130.7, 129.4, 129.4, 128.9, 128.8, 128.3, 126.9, $126.8,125.8,124.9,124.2,120.5,116.3,116.0,115.3,112.5,105.1,83.4,70.9,54.1,47.7,17.3$; IR (film) 3410 (broad), 1728, 1697, 1557, $1450 \mathrm{~cm}^{-1}$; HRMS (ESI) $\mathrm{m} / z$ calc'd for $\left[\mathrm{C}_{32} \mathrm{H}_{26} \mathrm{BrN}_{5} \mathrm{O}_{5}+\mathrm{H}\right]^{+}:$: 640.1196, found 640.1180.

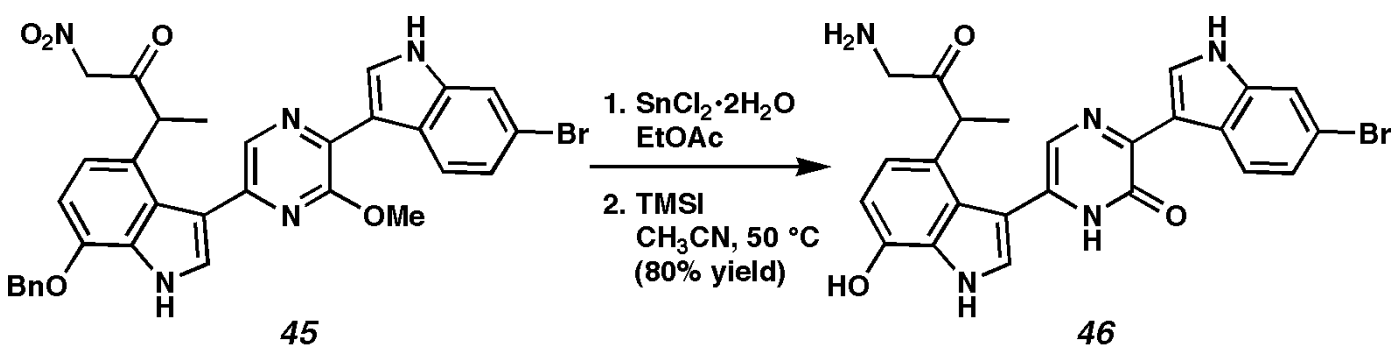

Aminoketone 46. To a solution of deprotected ketone $45(5.5 \mathrm{mg}, 0.0086 \mathrm{mmol})$ in EtOAc $(600 \mu \mathrm{L}$, deoxygenated by bubbling with argon for $1 \mathrm{~min})$, was added $\mathrm{SnCl}_{2} \cdot 2 \mathrm{H}_{2} \mathrm{O}(30$ $\mathrm{mg}, 0.13 \mathrm{mmol})$. The reaction vessel was equipped with a reflux condenser and heated at $80{ }^{\circ} \mathrm{C}$ for $3 \mathrm{~h}$. After cooling to $23{ }^{\circ} \mathrm{C}$, the solvent was removed under reduced pressure to leave an orange residue which was purified by reversed-phase filtration through a Sep-Pak column: first $10 \%$ acetonitrile containing $0.1 \%(\mathrm{wt} / \mathrm{v})$ TFA to remove salts, then $90 \%$ acetonitrile containing $0.1 \%(\mathrm{wt} / \mathrm{v})$ TFA to collect the crude product. After removal of solvent in vacuo, the compound was filtered through silica gel $\left(5: 1 \mathrm{CH}_{2} \mathrm{Cl}_{2} /\right.$ methanol eluent) to provide the reduced compound which was used without further purification.

To the crude aminoketone in acetonitrile $(700 \mu \mathrm{L})$ at $0{ }^{\circ} \mathrm{C}$, in a Schlenk tube, was added iodotrimethylsilane $(150 \mu \mathrm{L}, 1.05 \mathrm{mmol})$. The reaction mixture was heated at $50{ }^{\circ} \mathrm{C}$ for $2 \mathrm{~h}$, cooled to $0{ }^{\circ} \mathrm{C}$, then quenched with a saturated aqueous solution of sodium metabisulfite. The reaction mixture was purified by reversed-phase filtration through a Sep-Pak column: first $10 \%$ acetonitrile containing $0.1 \%(\mathrm{wt} / \mathrm{v})$ TFA to remove salts, then $90 \%$ acetonitrile containing $0.1 \%$ $(w t / v)$ TFA to collect the crude product. After removal of solvent under reduced pressure, the compound was further purified by reversed-phase HPLC. Concentration under reduced pressure 
provided the fully deprotected aminoketone $39\left(3.5 \mathrm{mg}, 80 \%\right.$ yield) as an orange/red oil: ${ }^{1} \mathrm{H}$ NMR (500 MHz, CD 3 OD) $\delta 8.75(\mathrm{~s}, 1 \mathrm{H}), 8.60(\mathrm{~d}, J=8.4 \mathrm{~Hz}, 1 \mathrm{H}), 7.67(\mathrm{~s}, 1 \mathrm{H}), 7.62(\mathrm{~d}, J=$ $1.7 \mathrm{~Hz}, 1 \mathrm{H}), 7.54(\mathrm{~s}, 1 \mathrm{H}), 7.27(\mathrm{dd}, J=8.5,1.8 \mathrm{~Hz}, 1 \mathrm{H}), 6.69(\mathrm{~d}, J=8.1 \mathrm{~Hz}, 1 \mathrm{H}), 6.63(\mathrm{~d}, J=$ $7.7 \mathrm{~Hz}, 1 \mathrm{H}), 4.15(\mathrm{q}, J=6.9 \mathrm{~Hz}, 1 \mathrm{H}), 3.64(\mathrm{~d}, J=1.7 \mathrm{~Hz}, 2 \mathrm{H}), 1.44(\mathrm{~d}, J=6.8 \mathrm{~Hz}, 3 \mathrm{H}) ;{ }^{13} \mathrm{C}$ NMR (125 MHz, $\left.\mathrm{CD}_{3} \mathrm{OD}\right) \delta$ 204.4, 157.4, 151.0, 145.3, 139.1, 132.6, 131.7, 129.0, 128.3, 127.7, 126.7, 126.1, 125.6, 124.9, 122.6, 121.0, 117.1, 115.5, 113.7, 108.5, 107.9, 46.8, 17.2; IR (film) 3140 (broad), 1671, 1200, $1140 \mathrm{~cm}^{-1}$; HRMS (ESI) $\mathrm{m} / z$ calc'd for $\left[\mathrm{C}_{24} \mathrm{H}_{20} \mathrm{BrN}_{5} \mathrm{O}_{3}+\mathrm{H}\right]^{+}$: 506.0828, found 506.0827.

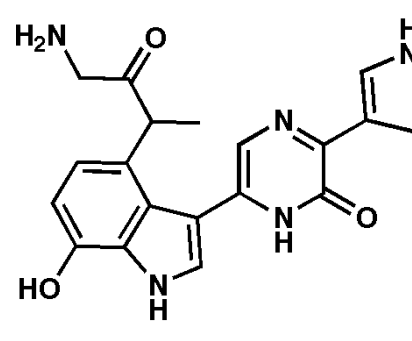

46

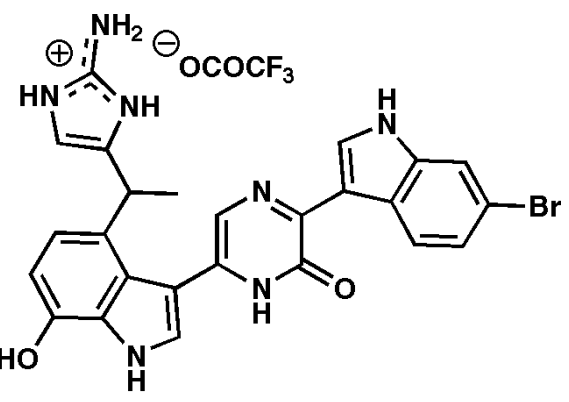

( \pm -Dragmacidin $D(5)$

Dragmacidin D (5). To a solution of aminoketone 46 (2 $\mathrm{mg}, 0.0039 \mathrm{mmol})$ in ethanol (700 $\mu \mathrm{L}$, deoxygenated by bubbling with argon for $5 \mathrm{~min}$ ) was added cyanamide (15 $\mathrm{mg}, 0.36$ mmol). The reaction vessel was purged with argon, sealed, and heated to $60{ }^{\circ} \mathrm{C}$ for $3 \mathrm{~h}$. After cooling to $23{ }^{\circ} \mathrm{C}$, the reaction mixture was purified by reversed-phase filtration through a Sep-Pak column: first $10 \%$ acetonitrile containing $0.1 \%(\mathrm{wt} / \mathrm{v})$ TFA to remove salts, then $60 \%$ acetonitrile containing $0.1 \%(\mathrm{wt} / \mathrm{v})$ TFA to collect the product. After removal of solvent under reduced pressure, dragmacidin $\mathrm{D}\left(\mathbf{5}, 1.8 \mathrm{mg}, 86 \%\right.$ yield) was isolated as an orange/red oil: ${ }^{1} \mathrm{H}$ NMR (600 $\left.\mathrm{MHz}, \mathrm{CD}_{3} \mathrm{OD}\right) \delta 8.74(\mathrm{~s}, 1 \mathrm{H}), 8.6(\mathrm{~d}, J=8.7 \mathrm{~Hz}, 1 \mathrm{H}), 7.62(\mathrm{~d}, J=1.8 \mathrm{~Hz}, 1 \mathrm{H}), 7.49(\mathrm{~s}, 1 \mathrm{H})$, 7.46 (s, 1H), 7.27 (dd, $J=8.2,1.8 \mathrm{~Hz}, 1 \mathrm{H}), 6.84$ (d, $J=8.2 \mathrm{~Hz}, 1 \mathrm{H}), 6.64(\mathrm{~d}, J=7.8 \mathrm{~Hz}, 1 \mathrm{H})$, $5.98(\mathrm{~s}, 1 \mathrm{H}), 4.35(\mathrm{q}, J=6.9 \mathrm{~Hz}, 1 \mathrm{H}), 1.52(\mathrm{~d}, J=7.3 \mathrm{~Hz}, 3 \mathrm{H}) ;{ }^{13} \mathrm{C}$ NMR $\left(125 \mathrm{MHz}, \mathrm{CD}_{3} \mathrm{OD}\right)$ $\delta 157.1,150.3,148.7,144.8,139.1,134.2,132.4,132.2,128.7,127.9,127.3,126.7,126.3,125.6$, 124.8, 120.2, 117.1, 115.4, 113.7, 110.2, 108.9, 107.3, 33.2, 20.8; IR (film) 3200 (broad), 1667, 1204, $1138 \mathrm{~cm}^{-1}$; UV $\lambda_{\max }(\mathrm{EtOH}) 216,274,389 \mathrm{~nm}$. After addition of 1 drop concentrated $\mathrm{HCl}$ 
to $1 \mathrm{~mL}$ cell: $\lambda_{\max }$ (EtOH) $219,277,460 \mathrm{~nm}$; HRMS (ESI) $m / z$ calc'd for $\left[\mathrm{C}_{25} \mathrm{H}_{20} \mathrm{BrN}_{7} \mathrm{O}_{2}+\mathrm{H}\right]^{+}$: 530.0940, found 530.0943. See Comparison ${ }^{1} \mathrm{H}$ NMR spectra: 


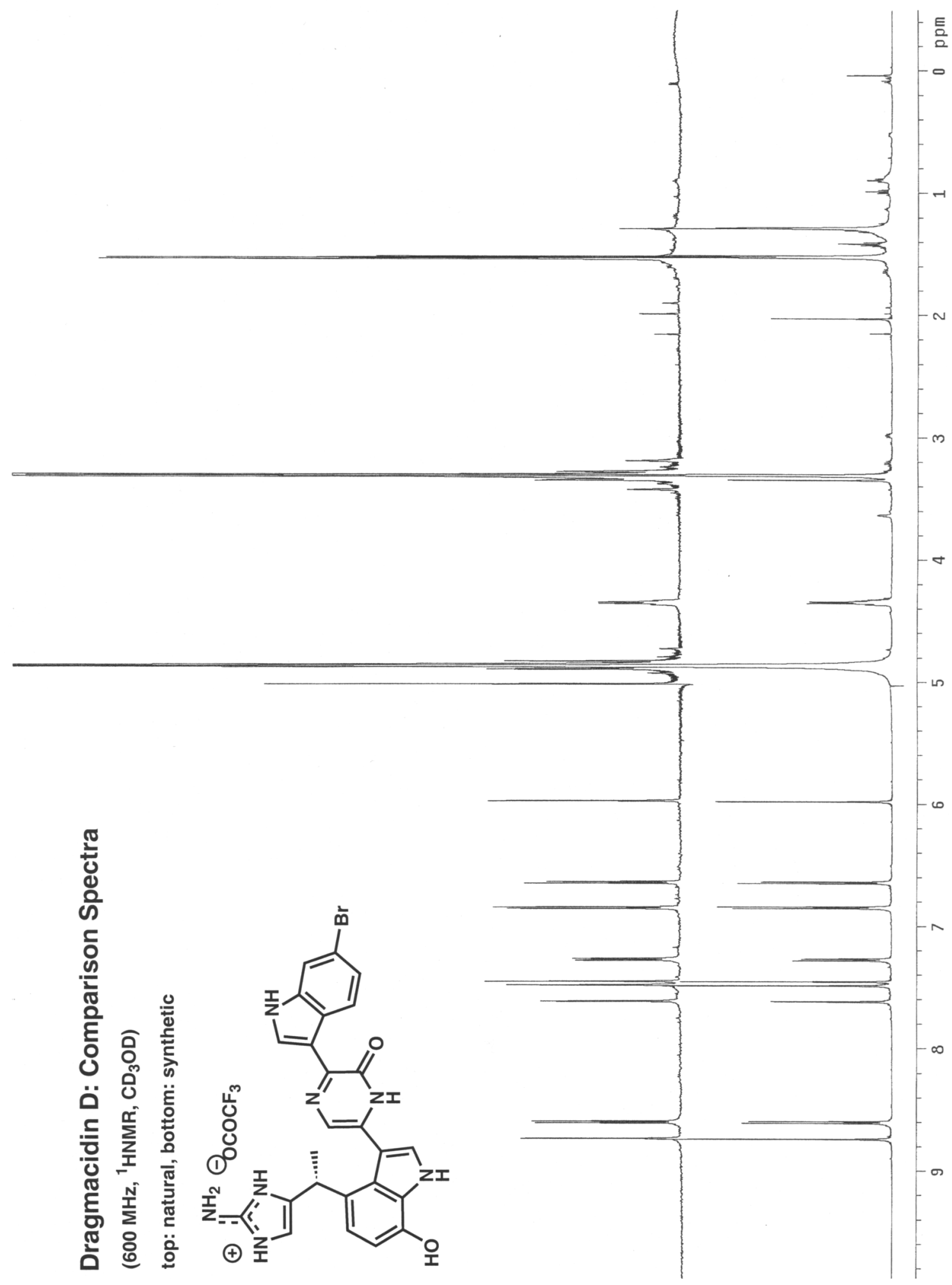

